\title{
Machine learning approach for predicting Fusarium culmorum and F. proliferatum growth and mycotoxin production in treatments with ethylene-vinyl alcohol copolymer films containing pure components of essential oils
}

\author{
Andrea Tarazona ${ }^{a}$, Eva M. Mateo ${ }^{a}$, José V. Gómez ${ }^{a}$, Rafael Gavara ${ }^{b}$, Misericordia Jiménez ${ }^{a}$, \\ Fernando Mateo ${ }^{c}$

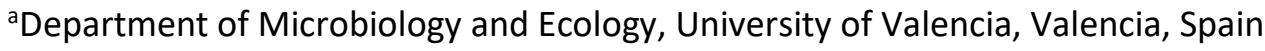

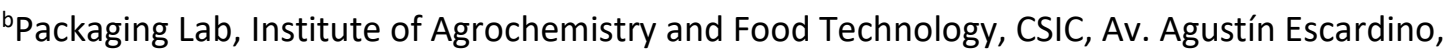 \\ 7, 46980 Paterna, Valencia, Spain \\ 'Department of Electronic Engineering, ETSE, University of Valencia, Valencia, Spain
}

\section{Abstract}

Fusarium culmorum and F. proliferatum can grow and produce, respectively, zearalenone (ZEA) and fumonisins (FUM) in different points of the food chain. Application of antifungal chemicals to control these fungi and mycotoxins increases the risk of toxic residues in foods and feeds, and induces fungal resistances. In this study, a new and multidisciplinary approach based on the use of bioactive ethylene-vinyl alcohol copolymer (EVOH) films containing pure components of essential oils (EOCs) and machine learning (ML) methods is evaluated. Bioactive EVOH-EOC films were made incorporating cinnamaldehyde (CINHO), citral (CIT), isoeugenol (IEG) or linalool (LIN). Several ML methods (neural networks, random forests and extreme gradient boosted trees) and multiple linear regression (MLR) were applied and compared for modeling fungal growth and toxin production under different water activity $\left(\mathrm{a}_{\mathrm{w}}\right)$ (0.96 and 0.99$)$ and temperature $\left(20\right.$ and $\left.28^{\circ} \mathrm{C}\right)$ regimes. The effective doses to reduce fungal growth rate $(G R)$ by 50,90 and $100 \%\left(E D_{50}, E D_{90}\right.$, and $\left.E D_{100}\right)$ of $E O C s$ in $E V O H$ films were in the ranges 200 to $>3330,450$ to $>3330$, and 660 to $>3330 \mu \mathrm{g} /$ fungal culture ( $25 \mathrm{~g}$ of partly milled maize kernels in Petri dish), respectively, depending on the EOC, $a_{w}$ and temperature. The type of EVOH-EOC film and EOC doses significantly affected GR in both species and ZEA and FUM production. Temperature also affected GR and $\mathrm{a}_{\mathrm{w}}$ only affected GR and FUM production of $F$. proliferatum. EVOH-CIT was the most effective film against both species and ZEA and FUM production. Usually, when the EOC levels increased, GR and mycotoxin levels in the medium decreased although some treatments in combination with certain $\mathrm{a}_{\mathrm{w}}$ and temperature values induced ZEA production. Random forest models predicted the GR of $F$. culmorum and $F$. proliferatum and ZEA and FUM production better than neural networks or extreme gradient boosted trees. The MLR mode provided the worst performance. This is the first approach on the ML potential in the study of the impact that bioactive EVOH films containing EOCs and environmental conditions have on F. culmorum and F. proliferatum growth and on ZEA and FUM production. The results suggest that these innovative packaging systems in combination with ML methods can be promising tools in the prediction and control of the risks associated with these toxigenic fungi and mycotoxins in food

\section{Keywords}

Fusarium culmorum, Fusarium proliferatum, zearalenone, fumonisins, machine learning methods, bioactive EVOH-films 


\section{Introduction}

Fusarium culmorum and F. proliferatum are two common phytopathogenic species that affect food in pre- and post-harvest worldwide. Fusarium culmorum (W.G. Smith) Saccardo is one of the main zearalenone (ZEA)-producing species, an estrogen-like compound that binds to the estrogen receptor and causes damage to germ cells and testicular structure (Yang et al., 2018). This species is frequently found in cereals and soybean, among others (Laraba et al., 2017; Wegulo et al., 2015). F. proliferatum (Matsushima) Nirenberg is one of the main fumonisin (FUM)-producing species. FUM are toxic compounds associated with leukoencephalomalacia in equine, hepatic and renal toxicity in rodents, pulmonary edema in pigs and esophageal cancer and neural tube defects in humans (Feijó Corrêa et al., 2018). The IARC has classified FUM, mainly fumonisin B1 (FB1), as possible carcinogenic compounds to humans (Group 2B) (Ostry et al., 2017). F. proliferatum is found in cereals (Cendoya et al., 2018; Gil-Serna et al., 2013; Stepien et al., 2011), soybean (Chang et al., 2015), date-palm trees (Saleh et al., 2017) or tomato (Gao et al., 2016), among others. So, ZEA and FUM, especially FB1 and fumonisin B2 (FB2), are mycotoxins widely distributed in food all over the world (Tarazona et al., 2020a; Waśkiewicz et al., 2012).

Although for a long time chemical antifungal agents are being used to control fungal growth and mycotoxin production in food (Magan et al., 2002; Mateo et al., 2013, Mateo et al., 2017a; Tarazona et al., 2020b), according to the Food and Agriculture Organization, about 1000 million metric tons of food is spoiled globally each year due to mycotoxins produced by storage molds (Bhat et al., 2010). This problem is exacerbated because consumers demand to minimize chemical additives to inhibit microbial growth in foods while maintaining quality, freshness, and safety. Therefore, new techniques are being developed. In this line, bioactive antimicrobial packaging systems are a possible option and an innovative method to provide increased food safety and quality (Nguyen Van Long et al., 2016). This is a technology that can be used simultaneously with others in the so-called hurdles technology (Suppakul et al., 2003).

In recent years, plant products such as essential oils (EOs) and their major bioactive compounds (EOCs), included in the Generally Recognized As Safe (GRAS) category, have been extensively studied as alternatives to synthetic chemicals and different EO formulations are currently assayed or used as food preservatives (da Cruz Cabral et al., 2013; Gómez et al., 2018; Prakash et al., 2015). EOs are produced by more than 17,000 aromatic plant species commonly belonging to angiospermic families Lamiaceae, Rutaceae, Myrtaceae, Zingiberaceae and Asteraceae (Prakash et al., 2015). EO formulations can be added to food directly or by slow release from packaging materials. Among the first, fumigation is one of the best methods to prevent microbial contamination during storage with no/less residual effect. Direct addition of EOs to food causes an immediate reduction of microbial populations. However, if the EO residues are rapidly depleted due to its volatility the recovery of injured cells or the growth of cells that were not destroyed by direct addition may not be prevented. Antimicrobial packaging is a technology that inhibits or retards the proliferation of microorganisms in foods, thus extending the shelf life of the product (Muriel-Galet et al., 2013; Zhang et al., 2015). Thus, the application of antimicrobial films containing EOs permits migration of the antimicrobial to the coating surface and provides a continuous antimicrobial effect on the food during extended exposure (Ribeiro-Santos et al., 2017).

\section{Materials and methods}

\subsection{Film preparation}


Ethylene-vinyl alcohol copolymer with $29 \%$ ethylene molar content (EVOH-29) was supplied by The Nippon Synthetic Chemical Industry Co., Ltd. (Osaka, Japan). Trans-cinnamaldehyde (3phenyl-2-propenal) (CINHO), linalool (LIN), isoeugenol (IEG) and citral (CIT) were supplied by Sigma-Aldrich (Barcelona, Spain). The films were made as described by Mateo et al. (2017a). Briefly, films of EVOH with an initial content of 1, 2, 5 and $10 \%$ of EOC were prepared by casting in an oven at $75^{\circ} \mathrm{C}$ for $15 \mathrm{~min}$. The final content of EOC was determined by thermal desorption-gas chromatography as described by Cerisuelo et al. (2012). The response of the gas chromatograph was calibrated by measuring polyethylene and polypropylene samples with known amounts of CINHO, CIT, IEG and LIN. The additive content is expressed as weight percentage of the compound over dry polymer weight. All the obtained EVOH-29 films containing pure components of plant essential oils were colorless, transparent and continuous. The final contents for CINHO, CIT, IEG or LIN found in the films, were $(0.37 \pm 0.06) \%$, $(0.74 \pm 0.12) \%,(1.85 \pm 0.25) \%$ and $(3.7 \pm 0.5) \%$, w/w of dry polymer, for the films prepared at $1,2,5$ and $10 \%$, respectively, regardless of the EOC type. The grammage of the bioactive EVOH films was $(1.79 \pm 0.02) \mathrm{mg} / \mathrm{cm}^{2}$. The obtained films were labelled as EVOH-CINHO, EVOH-CIT, EVOH-IEG or EVOH-LIN.

\subsection{Standards and reagents}

Mycotoxin standards of ZEA, $\mathrm{FB}_{1}$, and $\mathrm{FB}_{2}$ were supplied by Sigma (Sigma-Aldrich, Alcobendas, Spain). Formic acid and ammonium formate were purchased from Panreac (Castellar del Valles, Spain). Acetonitrile and methanol were purchased from J.T. Baker (Deventer, the Netherlands). All solvents were LC grade. Pure water was obtained from a Milli-Q apparatus (Millipore, Billerica, MA, USA) and was used when water was required.

\subsection{Inoculum preparation}

F. culmorum strain Fc019 and F. proliferatum strain Fp06 previously isolated from Spanish maize were used. The strains are held in the Mycology and Mycotoxins Group Culture Collection (Valencia University, Spain). The strains were grown on maize extract medium (MEM) ( $3 \% \mathrm{w} / \mathrm{v}$ of ground maize kernels $+2 \% \mathrm{w} / \mathrm{v}$ agar in pure water). MEM was sterilized in autoclave $\left(115^{\circ} \mathrm{C}, 30 \mathrm{~min}\right)$ and transferred into Petri dishes. $5 \mu \mathrm{L}$ of a spore suspension $\left(1 \times 10^{6}\right.$ spores $/ \mathrm{mL}$ ) of each fungal species was inoculated on the center of the plates and incubated at $30{ }^{\circ} \mathrm{C}$ for 7 days. Conidia of these fresh cultures were used to prepare inocula for further experiments. Before each experiment, a suspension of spores containing $1 \times 10^{6}$ conidia/mL, (a Thoma cell chamber counting was used), was prepared in sterile pure Milli-Q water containing Tween 80 (0.005\%) (Tarazona et al., 2020b).

\subsection{Preparation of fungal culture with different bioactive films and $a_{w}$ levels}

The assays with bioactive EVOH-EOC films were carried out in partially milled maize kernels (25 g) (pieces of 1-3 mm) as indicated by Tarazona et al. (2018). Partially milled maize, previously analyzed to ensure they had undetectable levels of $Z E A, F_{1}$ and $F B_{2}$, was placed in Erlenmeyer flasks and autoclaved for $20 \mathrm{~min}$ at $121^{\circ} \mathrm{C}$. Flasks containing milled maize destined to measure water activity $\left(a_{w}\right)$ values only were run at the same time. Then, milled maize was adjusted to 0.99 or $0.96 \mathrm{a}_{\mathrm{w}}$ by adding sterile pure water using specific curves. The $\mathrm{a}_{\mathrm{w}}$-values were measured with a Novasina RTD 502 equipment (Novasina GmbH, Pfäffikon, Switzerland) using controls of known $a_{w}$ values. The culture media were spread into 9-cm sterile Petri dishes. Then, circular pieces ( $8 \mathrm{~cm}$ diameter) of each bioactive EVOH film with final doses of $333,666,1665$ and $3330 \mu \mathrm{g}$ of each EOC per piece were attached on the base of the Petri dish lid with double sided adhesive cellophane. The final doses of EOC per piece of film were 
calculated as the product of content of each EOC in the film by grammage by film surface (Section 2.1). Preliminary experiments were carried out to choose a suitable range of concentrations for each EOC to be added to the $\mathrm{EVOH}$ films to obtain dose-response curves.

All media in Petri dishes, with and without EVOH-EOC films, were centrally inoculated with $15 \mu \mathrm{L}$ of a fresh conidia suspension $\left(1 \times 10^{6}\right.$ conidia/mL) of $F$. culmorum or F. proliferatum and were immediately capped and sealed with Parafilm $\mathrm{M}^{\circledR}$. The three replicates of inoculated Petri plates of the same treatment ( $a_{w}$ and type of film) were enclosed in sealed plastic containers together with beakers of a glycerol-water solution matching the same $a_{w}$ as the treatment to maintain a constant equilibrium relative humidity inside the boxes. Treatments and control cultures were incubated at 20 and $28{ }^{\circ} \mathrm{C}$ for 21 days in the darkness. All experiments were run in triplicate and repeated twice.

\subsection{Effect of treatments on fungal growth}

Growth in treatments and control cultures was monitored every day during the incubation period by measuring two diameters of the growing colonies at right angles with the help of a magnifying glass and calculated. Colony radius was calculated as the sum of the two diameters divided by four. The radial GR ( $\mathrm{mm} /$ day) was calculated as the slope of the line obtained by linear regression of mean colony radius versus time (only the linear part of this plot was used). The doses of EOC in the EVOH film/plate necessary for $50 \%, 90 \%$ and $100 \%$ growth inhibition $\left(E_{50}, E D_{90}\right.$, and $\left.E D_{100}\right)$ (the last $E D$ is also known as minimal inhibitory concentration, $M I C$ ) were estimated when possible from the plots of GR vs EVOH-EOC dose. All experiments were performed in triplicate and repeated twice.

\subsection{Effect of treatments on mycotoxin production}

\subsubsection{Mycotoxin determination in milled maize cultures}

At the end of the incubation period ( 21 days), regardless of the size reached by the fungal colony, the total of culture (substrate plus fungal biomass) (approx. $25 \mathrm{~g}$ ) in controls and treatments was removed from Petri dishes and dried at $45{ }^{\circ} \mathrm{C}$ until constant weight was achieved. Then they were finely milled, homogenized and analyzed for ZEA ( $F$. culmorum cultures) and $\mathrm{FB}_{1}$ and $\mathrm{FB}_{2}$ (F. proliferatum cultures) following the methodology described by Tarazona et al. (2020b). A portion of milled culture (1-2 g) was placed into a Falcon tube and mycotoxins were extracted shaking with $8 \mathrm{~mL}$ acetonitrile/water/formic acid (80:19:1, v/v/v) in orbital shaker for $1 \mathrm{~h}$. After centrifugation $(4260 \mathrm{~g}, 5 \mathrm{~min}), 2 \mathrm{~mL}$ of the supernatant was filtered through $0.22-\mu \mathrm{m}$ PTFE syringe filter. Extracts from cultures were diluted $(1: 2, \mathrm{v} / \mathrm{v})$ with the same solvent before injection into the chromatographic system. When mycotoxin levels were too high for the linear calibration range, the extracts were appropriately diluted with the same solvent mixture and injected again.

For calibration purposes, matrix-matched calibration was performed. Mycotoxin standard solutions were obtained by dissolution of pure standards of $\mathrm{ZEA}, \mathrm{FB}_{1}$ and $\mathrm{FB}_{2}$ in acetonitrile:water (1:1, v/v) and further dilution with acetonitrile/water/formic acid (80:19:1, $\mathrm{v} / \mathrm{v} / \mathrm{v}$ ). Calibration standards were prepared by addition of variable volumes of ZEA, $\mathrm{FB}_{1}$ or $\mathrm{FB}_{2}$ standard solutions to extracts of non-inoculated milled maize (blanks) prepared as indicated above for cultures. Blanks were previously analyzed and found to contain no detectable amount of the target mycotoxins. Aliquots of filtered blank extracts were diluted $(1: 2, \mathrm{v} / \mathrm{v})$ with the same extracting solvent. Then, aliquots of $2 \mathrm{~mL}$ of the diluted extract were evaporated to dryness under a stream of $\mathrm{N}_{2}$, added with appropriate volumes of standard 
solutions and brought to a volume of $2 \mathrm{~mL}$ with acetonitrile/water/formic acid (80:19:1, v/v/v) to provide the desired concentrations. The calibration range in the diluted extract was $0.05-$ $5.0 \mu \mathrm{g} / \mathrm{mL}$ for $\mathrm{FB}_{1}$ and for $\mathrm{FB}_{2}$ and $0.045-4.0 \mu \mathrm{g} / \mathrm{mL}$ for ZEA. Calibration lines were obtained by weighted linear regression (weighting factor $1 / x$ ) of the areas of peaks associated to the quantifier ion of each analyte vs mycotoxin concentration.

The method was validated in-house by recovery assays and replication of analyses as described by Tarazona et al. (2020a) with some modifications related to mycotoxin concentrations and spiking levels in recovery assays. The concentrations of mycotoxins in spiked blanks were in the ranges $2.5-200 \mu \mathrm{g} / \mathrm{g}$ for $Z E A, 5-150 \mu \mathrm{g} / \mathrm{g}$ for $\mathrm{FB}_{1}$ and 3-50 $\mu \mathrm{g} / \mathrm{g}$ for $\mathrm{FB}_{2}$.

\subsubsection{UPLC-MS/MS conditions}

The separation and MS/MS conditions were as reported by Romera et al. (2018). The UPLCMS/MS system consisted of an ACQUITY UPLC ${ }^{\mathrm{TM}}$ system (Waters, Manchester, UK), provided with an electrospray ionization interface (ESI) that was used in positive ion mode (ESI+). Separation was performed at $25^{\circ} \mathrm{C}$ in an ACQUITY UPLC BEH C18 column $(50 \times 2.1 \mathrm{~mm}, 1.7 \mu \mathrm{m}$ particle size) (Waters). The mobile phase was a time-programmed combination of water containing ammonium formate $(0.15 \mathrm{mmol} / \mathrm{L})$ and formic acid $(0.1 \%)$ (solvent $\mathrm{A})$ and methanol (solvent B) at a flow-rate of $0.37 \mathrm{~mL} / \mathrm{min}$. A volume of $30 \mu \mathrm{L}$ was injected. MS/MS detection was performed by an ACQUITY TQD tandem quadrupole mass spectrometer (Waters Co., Milford, MA, USA). Two product ions (1 quantifier, 1 qualifier) were monitored for each mycotoxin. Masslynx 4.1 $1^{\mathrm{TM}}$ chromatographic software (Waters, Manchester, UK) was used for data acquisition and processing. Retention time of the target peaks (quantifier and qualifier) in multiple reaction monitoring (MRM) chromatograms was used for identification.

\subsection{Data analysis}

\subsubsection{Analysis of variance}

Data were analyzed by multifactor analysis of variance (ANOVA) using Statgraphics Centurion XV.II statistical package (StatPoint, Inc., VA, USA). Post-hoc Tukey's honestly significant difference (Tukey's HSD) ( $\alpha=0.05$ ) was usually used to find homogenous groups when significant differences among the levels were revealed by ANOVA. GR and concentrations values were transformed as $\log (x+1)$ where $x$ was the raw value of the variable to reduce spread.

\subsubsection{ML model development}

The compared ML methods for modeling fungal GR and mycotoxin production in cultures were NN (neuralnet library) (Günther and Fritsch, 2010), RF (randomForest library) (Liaw and Wiener, 2002) and XGBoost (XGBTree library) (Chen and Guestrin, 2016). The used methodology has been described previously (Tarazona et al., 2020a). For comparison purposes, a multiple linear regression (MLR) model was also developed for every dependent output variable (outcome). The equations have the general form in Eq. (1).

Ethylene-vinyl alcohol copolymer containing $29 \%$ mol ethylene (EVOH-29) is a packaging material commonly used to provide high oxygen barrier properties. EVOH materials have been used as matrices for the development of active packaging systems, where the polymer protects the active agents during storage and triggers their activity on exposure to a humid 
environment (the food product) (Catalá et al., 2016; Muriel-Galet et al., 2012). These properties combined with appropriated EOC, could make EVOH a highly suitable material for control of toxigenic fungi (aerobic organisms) and their associated mycotoxins (Mateo et al., 2017a; Tarazona et al., 2018) in food and feed.

The growth of toxigenic fungi, including F. culmorum and F. proliferatum, and mycotoxin production, mainly depends on temperature, water activity (aw)/relative humidity ( $\mathrm{RH}$ ) and possible treatments with antifungal agents (Magan et al., 2002, Magan et al., 2003). These factors have also been considered in studies on the effectiveness of bioactive films in controlling fungal growth and mycotoxin production (Mateo et al., 2017a; Tarazona et al., 2018).

Machine learning (ML) methods involve many disciplines, such as probability theory, statistics, approximation theory, etc. and supervised ML can be used in prediction analysis (Qu et al., 2019). Predictive models based on ML methods can be innovative tools for the management of toxigenic fungi and mycotoxin production and can help to set preventive treatments. Among such ML methods, artificial neural networks (NN) have been used to predict fungal growth (Panagou et al., 2007; Panagou and Kodogiannis, 2009) or mycotoxin production by fungi (Mateo et al., 2009, Mateo et al., 2011a). Random forests (RF) are ensembles of decision trees. They are supervised ML models that have also been applied in mycology for prediction (Breiman, 2001; Meher et al., 2019). Extreme gradient boosted trees (XGBoost) have been applied in a previous mycological study (Tarazona et al., 2020a).

The aims of this study were: (1) to evaluate the effect of EVOH-29 films incorporating cinnamaldehyde (CINHO), citral (CIT), isoeugenol (IEG) or linalool (LIN) (pure components of plant essential oils) to control the growth of F. culmorum and F. proliferatum in partially milled maize under different environmental conditions, (2) to investigate the effects of these treatments and environmental conditions on ZEA production by F. culmorum and on FB1 and FB2 production by F. proliferatum, (3) to design ML methods able to predict the growth rates (GR) of these fungi and mycotoxin production under all the assayed conditions.

$$
Y=\beta_{0}+\sum_{i=1}^{n} \beta_{i} X_{i}+\varepsilon(i=1,2, \ldots n)
$$

where $Y$ denotes the output variable, $\boldsymbol{B}_{0}$ is an independent term (intercept), $\boldsymbol{b}_{i}$ are the estimates of coefficients for $X_{i}$, which are the independent variables (predictors), $\varepsilon$ is the error term (unknown) and $n$ is the number of predictors. The software used was $R$ and the 'classification and regression training' (caret) package (Kuhn, 2016). The two species were treated separately. The input variables were four (temperature, $a_{w}, E O C$ type and EOC concentration). The output variables were a) GR of $F$. culmorum, b) production of ZEA by $F$. culmorum, c) GR of $F$. proliferatum, d) production of $\mathrm{FB}_{1}$ by $F$. proliferatum and e) production of $\mathrm{FB}_{2}$ by $F$. proliferatum. The output values were the average of six measurements to minimize uncertainty. Some preprocessing of variables was performed. For mycotoxin outputs, the natural logarithm of average mycotoxin concentration plus 1 was computed to avoid problems with undetectable concentrations. Categorical variables were recoded into a set of separate binary variables (indicator or dummy variables). Such recoding (dummy coding) was done 
automatically by the statistical software R. For a variable with $j$ levels, $j-1$ dummy variables were created to avoid multicollinearity. The level that is not shown is the reference level; the coefficients of the other levels are measured with respect to the reference level. For the ML models, every data set ( 80 per output variable) was randomly split into training $(75 \%)$ and test $(25 \%)$ sets. The training set was used to build and validate the models and the test set was let aside to evaluate its performance on independent external data, not previously shown to the models. MLR is a linear regression method and does not require a time-consuming procedure for parameter optimization; the coefficients are chosen to minimize the sum of squares of the residuals (least squares method). The metric used for performance evaluation of the ML methods was the averaged root mean square error (RMSE) for training over 10-fold cross-

validation. Cross-validation is a resampling procedure used to evaluate ML models on a limited data sample. It generally produces a less biased/optimistic estimate of the model skill than other methods. In 10-fold cross-validation, the data is divided into 10 subsets. A holdout method is repeated 10 times, such that each time, one of the 10 subsets is used as the validation set and the other 9 subsets are put together to form a training set. Thus, all observed values in the train subset are used for both training and validation, and each observation is used for validation exactly once. The error estimation (RMSE) is averaged over all 10 trials, which decreases bias because most of the data are used for fitting, and reduces variance because most of the data are used in validation set. The ML models were tuned to optimize their performance for each one of the tasks, according to their most significant parameters (Table 1 ). The $\mathrm{R}^{2}$ (coefficient of determination) values were also determined for each model and averaged over all ten-fold cross-validation. All predictive models were tested and compared against a test dataset ( $25 \%$ of the overall dataset) not used to train/crossvalidate the models. The RMSE and $\mathrm{R}^{2}$ values for these sets were obtained.

\section{Results}

\subsection{Antifungal activity of bioactive EVOH-EOC films}

Circular, easily visible and measurable colonies were observed on all fungal cultures of the present study when growth occurred. Growth delay was 2-4 days depending on culture conditions. The radial GR of $F$. culmorum and F. proliferatum colonies vs doses in control and treatments under the different conditions assayed are shown in Fig. 1, Fig. 2. No significant differences in radial GR were found in control cultures without any film or with films without $E O C$, incubated under the same conditions (temperature and $a_{w}$ ). Therefore, the values shown in Fig. 1, Fig. 2 for control cultures of $F$. culmorum and F. proliferatum, respectively, are the average of these six replicates ( 3 replicates each). The two species grew faster at $28^{\circ} \mathrm{C}$ than at $20{ }^{\circ} \mathrm{C}$ and at $0.99 \mathrm{a}_{\mathrm{w}}$ than at $0.96 \mathrm{a}_{\mathrm{w}}$. Under the same set of conditions, the GR of $F$. culmorum was significantly higher than the GR of $F$. proliferatum. Thus, their behavior was analyzed separately. In treatments, ANOVA showed that the type of antifungal agent, EOC doses, and temperature significantly influence the GR $(p<0.05)$ of $F$. culmorum and $F$. proliferatum. The GR of $F$. proliferatum was also significantly influenced by $\mathrm{a}_{\mathrm{w}}$. The interactions antifungal agent $\times$ dose and temperature $\times a_{w}$ (for $F$. culmorum) and the interactions antifungal agent $\times$ dose and antifungal agent $\times$ temperature $\times a_{w}$ (for $F$. proliferatum) were significant $(p<0.05)$. Table 2 shows the homogeneous groups obtained by the post hoc Tukey's HSD test. The presence of more than one group is due to the existence of significant differences $(p<0.05)$. This test provided two homogeneous groups for the type of bioactive EVOH films against $F$. culmorum and the order of efficacy was EVOH-CIT $>$ EVOH-IEG $\approx$ EVOH- 
$\mathrm{CINHO} \approx \mathrm{EVOH}$-LIN. For F. proliferatum, however, the Tukey's HSD test provided four homogeneous groups for the bioactive films (one for each film) and the order of efficacy was EVOH-CIT > EVOH-CINHO > EVOH-IEG > EVOH-LIN (Table 2).

Generally, for both fungi, the higher the dose the more efficient delay of fungal growth. The four doses were arranged in three homogeneous groups (3330-1665, 1665-666, and $333 \mu \mathrm{g} /$ Petri plate) by the Tukey's HSD test (Table 2). In the case of $F$. culmorum, the mycelium developed faster in controls than in treatments with EVOH-CIT, regardless of doses. With EVOH-IEG, the two high doses (3330-1665 $\mu \mathrm{g} /$ Petri plate) significantly decreased GR with respect to controls. With EVOH-LIN, only the highest dose significantly decreased GR with respect to controls (Tukey's HSD test). With EVOH-CINHO the Tukey's HSD test classified doses and controls in a homogeneous group. In F. proliferatum cultures, GR was significantly decreased by all the doses with respect to the controls, except for the lowest dose (333 $\mu \mathrm{g} /$ Petri plate) with EVOH-LIN.

Table 3 lists the effective doses (EDs) of the different EVOH-EOC films/Petri plate against $F$. culmorum and $F$. proliferatum under all assayed conditions. The EDs to reduce the GR of the fungi by 50,90 and $100 \%\left(E D_{50}, E D_{90}\right.$ and $\left.E D_{100}\right)$ were in the ranges 200 to $>3330,450$ to $>3330$, and 660 to $>3330 \mu \mathrm{g}$ of EOC/fungal culture, respectively, depending on the type of EVOH-EOC film, fungal species, $a_{w}$ and temperature. In general, with few exceptions, F. culmorum showed the greatest susceptibility to all active EVOH films at $20^{\circ} \mathrm{C}$ and $0.99 \mathrm{a}_{\mathrm{w}}$. However, the influence of environmental conditions on the susceptibility of $F$. proliferatum to EVOH-EOC films was not clear and depended on the particular film tested. Thus, for this species, the lowest EDs for EVOH-CIT films were recorded at $28^{\circ} \mathrm{C}$ and $0.99 \mathrm{a}_{\mathrm{w}}$, for EVOH-CINHO the combination $28^{\circ} \mathrm{C} / 0.96 \mathrm{a}_{\mathrm{w}}$ provided the lowest EDs. Overall, considering the two species and all the temperature/ $\mathrm{a}_{\mathrm{w}}$ combinations, the lowest EDs were registered in the EVOH-CIT treatments and the highest EDs in the EVOH-LIN treatments.

\subsection{Effect of bioactive EVOH-EOC films on mycotoxin production}

\subsubsection{Validation of the UPLC-MS/MS method}

For calibration lines, the $\mathrm{R}^{2}$ values were $>0.99$ and residuals were $<20 \%$. The average recoveries of $\mathrm{ZEA}, \mathrm{FB}_{1}$ and $\mathrm{FB}_{2}$ in spiked blanks were $95.3 \%, 95.2 \%$ and $96.0 \%$, respectively. The average relative standard deviation (RSDr) of recoveries ( 6 replicates per level) was $<5 \%$ for the three mycotoxins. Other validation parameters agree with those previously reported (Tarazona et al., 2020a).

\subsubsection{Production of mycotoxins}

In control cultures of $F$. culmorum and $F$. proliferatum without any film or with films without $E O C$, incubated under the same conditions (temperature and $a_{w}$ ) no significant differences on $Z E A$, and $F B_{1}$ and $F B_{2}$ levels were found. Thus, the levels of ZEA, shown in Fig. 3, and the levels of $\mathrm{FB}_{1}$ and $\mathrm{FB}_{2}$, shown in Fig. 4 , for control cultures of $F$. culmorum and $F$. proliferatum are the average of the six replicates ( 3 replicates each).

ANOVA revealed that the main factors EVOH-EOC type, EOC dose and temperature significantly influenced ZEA production by F. culmorum $(p<0.05)$ while $\mathrm{a}_{\mathrm{w}}$ did not. Two first order interactions (EVOH-EOC type $\times$ dose and EVOH-EOC type $\times$ temperature) were significant. The post-hoc Tukey's HSD test $(\alpha=0.05$ ) (Table 4 ) discriminated two homogeneous groups: one composed of EVOH-CIT and EVOH-IEG treatments and another group composed of 
EVOH-CINHO and EVOH-LIN treatments. ZEA concentration decreased with increasing dose. The Tukey's HSD test included each dose in a homogeneous group and there was not overlapping (Table 4). Although ZEA levels were higher at $0.99 a_{w}$ than at $0.96 a_{w}$, the differences were not significant $(p>0.05)$. Generally, ZEA production in controls was significantly higher than in treatments. Interestingly, ZEA levels in treatments at low LIN doses, $20^{\circ} \mathrm{C}$ and $0.96 \mathrm{a}_{\mathrm{w}}$ exceeded the levels reached in controls (Fig. 3). Correlation between $\log [C(Z E A)+1]$ and $\log (G R+1)$, where $C(Z E A)$ is concentration of ZEA, was significant $(R=0.700, p<0.0001)$. The $R^{2}$ value $(0.4905)$ indicates that the model obtained by linear regression explains $49.05 \%$ of the variability in the predicted variable.

According to multifactor ANOVA the main factors EVOH-EOC type, EOC dose and $\mathrm{a}_{\mathrm{w}}$ significantly influenced $\mathrm{FB}_{1}$ production by $F$. proliferatum. The interactions $\mathrm{EVOH}-\mathrm{EOC}$ type $\times$ dose, EVOH-EOC type $\times$ temperature, and temperature $\times a_{w}$ were significant. The Tukey's HSD test placed the film types in two homogeneous groups: one composed of EVOH$\mathrm{CIT}$ and EVOH-CINHO and another group composed of EVOH-IEG and EVOH-LIN (Table 4). The same test clustered the EOC dose in two different groups: 1) 3330, 1665, and $666 \mu \mathrm{g} /$ Petri plate and 2) $333 \mu \mathrm{g} /$ Petri plate (Table 4). $\mathrm{FB}_{1}$ level was significantly higher at $0.96 \mathrm{a}_{\mathrm{w}}$ than at $0.99 \mathrm{a}_{\mathrm{w}}$. There were significant differences on $\mathrm{FB}_{1}$ level between controls and treatments under the same conditions. The factors EVOH-EOC type and EOC dose significantly influenced $\mathrm{FB}_{2}$ production but temperature and $\mathrm{a}_{w}$ did not. The homogeneous groups depicted by Tukey's HSD test are shown in Table 4. Considering all treatments, there was a significant positive correlation between $\log \left[\mathrm{C}\left(\mathrm{FB}_{1}\right)+1\right]$ and $\log (\mathrm{GR}+1)$, where $\mathrm{C}\left(\mathrm{FB}_{1}\right)$ is concentration of $\mathrm{FB}_{1}$, $(R=0.828, p<0.0001)$. The $R^{2}$ value $(0.686)$ indicates that the linear model obtained by regression can explain $68.6 \%$ of the variability in the predicted variable.

\subsection{ML methods applied to prediction of fungal growth and mycotoxin production}

Table 5 summarizes the characteristics of the best ML models found and their RMSE and $\mathrm{R}^{2}$ values. As previously explained, the goal was to obtain the minimum average RMSE value for training with cross-validation but also secondarily maximizing $\mathrm{R}^{2}$.

For NN (single-hidden-layer perceptron) modeling, the optimal model settings were selected using the lowest RMSE value for training with cross-validation. Architectures with 5, 10 or 20 nodes in the hidden layer and a decay value of $0.1,0.2$ or 0.3 were essayed (Table 5 ). The models giving the lowest RMSE values usually provided the maximum $\mathrm{R}^{2}$ values.

For RF, the ntree parameter was fixed to 500 for all the output variables and the only parameter to modify was the number of randomly selected predictors to sample every time a tree splits (mtry). There were four input predictors (temperature, $\mathrm{a}_{w}$, type of EVOH-EOC film, and $E O C$ dose) so the tuning range of values was mtry $=[2,3,4]$. The performance was best for $m$ try $=4$, i.e. using all input predictors (Table 5). The obtained average RMSE was lower than for the NN or XGBoost. Thus, RF showed the overall best performance for predicting fungal GR, and ZEA and FUM production. This was further confirmed with the test set.

XGBoost models have the largest number of parameters to tweak. Hence, searching for optimal parameters takes more time than for NN and RF models. Nevertheless, given the reduced size of data sets, the training time for all the combinations listed in Table 1 is assumable. The best results were obtained from models with the parameter values given in Table 5. Some parameter values given in Table 1 were let constant (for example, nrounds $=150$, as it was high enough to achieve convergence in all simulations, gamma $=0$, as increasing it makes the algorithm more conservative, colsample_bytree $=1$ (default), 
min_child_weight $=0.5$ ). The optimal values for the variable parameters were max_depth (which controls the maximum number of nodes allowed from the root to the farthest leaf of a tree) $=2,3$ or 4 (depending on the output variable), eta (learning rate) $=0.1$ (except 0.2 for $F$. proliferatum $\mathrm{GR}$ and 0.3 for $\mathrm{FB}_{1}$ production), and subsample rate (randomly sample a portion of the training data prior to growing trees at every boosting iteration) $=1$ (except 0.75 for $F$. culmorum GR) (Table 5). The best XGBoost models produced minimal RMSE values for GR that were always higher than the minimal RMSE values obtained with the best NN or RF models designed. The $R^{2}$ values for the best XGBoost models were lower than the values obtained for the best NN or RF models (except for ZEA production and F. proliferatum GR). When comparing the relative accuracy of the models for predicting the GR of both species it can be seen (Table 5) that the RMSE were lower and the $\mathrm{R}^{2}$ were higher for $F$. proliferatum than for $F$. culmorum. For MLR, an equation was obtained by least squares regression using the $75 \%$ of the dataset for each of the five outcomes. These equations included an independent term (intercept) and the coefficient estimates for the predictor dummy variables.

Overall, the best performance for modeling fungal growth and mycotoxin production was obtained using RF models, although differences with other tested ML models were not high; for example, regarding ZEA production, the XGBoost model provided a training RMSE of 1.041, very near to the value of 1.032 given by the RF model (Table 5 ). They can be considered identical.

The results of the application of these models to the same dataset used for testing (not used for training) appear in Table 6. The RMSE values were always higher for the test set than for training, and the RF models achieved the best (lowest) RMSE values. However, similar RMSE values were computed for the RF and XGBoost models in the case of $\mathrm{FB}_{2}$. For the test set, the MLR models produced the highest RMSE values and the lowest $R^{2}$ values (Table 6 ). The relative performance of NN and XGBoost changed depending on the selected outcome. Fig. 5 shows the scatter plots of predicted versus observed values for the RF and MLR models applied to the same test sets for GR of both F. culmorum and F. proliferatum, and production of ZEA, $\mathrm{FB}_{1}$ and $\mathrm{FB}_{2}$. Negative predicted values obtained with the MLR models have no sense; they were considered as zero. A feature of RF is that there are not predictions (extrapolations) out of the bounds of the observed output values. RMSE values can only be used to compare models on a given outcome, not among them. For mycotoxins the computed outputs were $\ln (C(Z E A)+1)$, $\ln \left(C\left(\mathrm{FB}_{1}\right)+1\right)$ or $\ln \left(\mathrm{C}\left(\mathrm{FB}_{2}\right)+1\right)$, where In denotes natural logarithm and $\mathrm{C}(\mathrm{ZEA}), \mathrm{C}\left(\mathrm{FB}_{1}\right)$ and $\mathrm{C}\left(\mathrm{FB}_{2}\right)$ are concentrations of $\mathrm{ZEA}, \mathrm{FB}_{1}$ and $\mathrm{FB}_{2}$, respectively, as shown in Fig. 5.

\section{Discussion}

In the present study, the antifungal activity of EVOH-CINHO, EVOH-CIT, EVOH-IEG and EVOHLIN films in vapor phase against $F$. culmorum and $F$. proliferatum under different regimes of $a_{w}$ and temperature in partly milled maize grains was tested. The effect of these treatments on ZEA production by $F$. culmorum and $\mathrm{FB}_{1}$ and $\mathrm{FB}_{2}$ production by $F$. proliferatum was evaluated. Likewise, the impact of possible interactions between environmental conditions and treatments (type of bioactive film and doses) on fungal growth and mycotoxin biosynthesis was also analyzed. In the present study, the inhibitory doses or effective doses $E D_{50}, E D_{90}$, and $E D_{100}$ were studied. These parameters are usually given to describe the response of filamentous fungi to antifungal agents and they permit to perform comparative studies (Marín et al., 2013; Mateo et al., 2011b, Mateo et al., 2013, Mateo et al., 2017a, Mateo et al., 2017b; Sharma et al., 2017). 
No previous studies on F. culmorum and F. proliferatum growth and ZEA and FUM production, respectively, in treatments with active $\mathrm{EVOH}$ films containing $\mathrm{EO}$ or EOC have been reported so far. Lack of such reports hinders a critical and comparative discussion of the results. However, previous results have shown that growth of some Fusarium spp. may be inhibited by plant EOs (da Cruz Cabral et al., 2013). In most of the reports, EOs are included within the culture medium. Thus, Sharma et al. (2017) studied the effect of clove, lemon grass, mint and eucalyptus EOs against $F$. oxysporum f. sp. lycopersici and they found MIC values of 31.25, $62.5,125$ and $500 \mathrm{ppm}$, respectively, and fungicidal action at 125, 250 and $500 \mathrm{ppm}$, respectively. The lowest $\mathrm{IC}_{50}$ (50\% inhibitory concentration) values were recorded for clove EO (18.22 ppm) followed by lemongrass (24.25 ppm), mint (60.05 ppm) and eucalyptus (207.86 ppm), respectively. The inhibitory effect of cinnamon, citral, Litsea cubeba oil, clove, eucalyptus, anise, spearmint and camphor oils on $F$. verticillioides was investigated by Xing et al. (2014) and the most effective of them was cinnamon oil. The MICs of cinnamon oil (85\% cinnamaldehyde), natural cinnamaldehyde (95\%), and synthetic cinnamaldehyde (99\%) were 60,50 , and $45 \mathrm{~mL} / \mathrm{L}$, respectively. Hashem et al. (2010) using the agar diffusion method found that EOs extracted from cumin, basil and geranium have a significant antifungal activity against F. oxysporum, F. solani, F. moniliforme (F. verticillioides), F. dimerum, F. equiseti and $F$. lateritium. The most effective EO was cumin oil, the diameter of the fungal growth inhibition zone ranged from 2.17 to $0.00 \mathrm{~cm}$ depending on the fungal species and doses. The efficacy of clove oil nanoemulsions on Fusarium growth and mycotoxin production during malting process was explored by Wan et al. (2020). They observed that $1.5 \mathrm{mg}$ clove oil/g has a positive influence on barley grain germination and in the decrease in DON levels and fungal biomass. They suggest the potential application of EO nanoemulsion during the malting process. Dambolena et al. (2010) reported that the oil of Ocimum gratissimum with high content in eugenol (95.5\%-70.1\%) have high effectivity (MIC $0.3 \mu \mathrm{L} / \mathrm{mL}$ ) against $F$. verticillioides and it induces a significant inhibitory effect on $\mathrm{FB}_{1}$ production with respect to control $(p<0.05)$. Their results showed a direct relationship between the inhibitory effects of $E O$ on fungal growth and $\mathrm{FB}_{1}$ production. Seseni et al. (2015) found that lemongrass ( $84.8 \%$ citral), clove ( $88.3 \%$ of eugenol) and thyme (63.1\% thymol and $21.3 \%$ linalool) oils were able to control the mycelial growth of $F$. oxysporum and $F$. circinatum at levels of 300-700 $\mu \mathrm{L} / \mathrm{L}$ of medium. Vilaplana et al. (2018) assayed in vitro thyme, mint, rosemary and lavender oils against $F$. verticillioides on PDA. They found that thyme oil was the best EO to control mycelial growth. The results of the in vivo test showed the reduction of the severity of $F$. verticillioides on pineapples treated with $1000 \mu \mathrm{L} / \mathrm{L}$, after 21 days at $8{ }^{\circ} \mathrm{C}$ plus 7 days of shelf-life at $20^{\circ} \mathrm{C}$. They suggested that thyme oil might potentially be used to control fusariosis in pineapples during postharvest, without negative effects on its physicochemical and sensory qualities. The possible use of EOs in the control of Fusarium spp. is not limited to the fields of agriculture and food; for example, Manganyi et al. (2015) found that clove and thyme oils, as well as pure citral, eugenol and thymol at $500 \mu \mathrm{L} / \mathrm{L}$, significantly reduce radial growth colonies of $F$. oxysporum that form biofilms in contact lenses. Total inhibition of all isolates was obtained when the EOs were used at a concentration of $1000 \mu \mathrm{L} / \mathrm{L}$. They demonstrated that clove and thyme oils prevented mycelium cell attachment, biofilm development and caused total inhibition of biofilm formation on soft contact lenses. They suggested that the use of diluted clove and thyme oils could therefore be an option to prevent biofilm formation of soft contact lenses. Even if all cited reports illustrate the effectiveness of different EO against Fusarium spp., in none of them the EOCs included in our films have been assayed. In addition, the diversity of used methodologies does not permit a comparative critical study. However, in recent studies with relevant aflatoxigenic and ochratoxigenic Aspergillus spp. using EVOH-EOC films and the 
methodology applied in the present study, similar results were obtained (Mateo et al., 2017a; Tarazona et al., 2018). Therefore, it can be concluded that EOC from plants included in different packaging systems such as EVOH films, coatings, paints, etc., which facilitate their controlled release, can be an excellent tool for the prevention and control of toxigenic fungi and mycotoxins in food, including cereals, during silage, transportation or marketing.

The effect of films containing EOCs on ZEA production by F. culmorum and $\mathrm{FB}_{1}$ and $\mathrm{FB}_{2}$ production by $F$. proliferatum has not been reported to date. Most of the studies have focused on the production of aflatoxins or ochratoxin A (OTA) by Aspergillus spp. (Mateo et al., 2017a; Nguyen Van Long et al., 2016; Tarazona et al., 2018). However, some previous studies on the effect of different EOs incorporated into culture media against closely related species, such as F. graminearum or F. verticillioides have been published. Thus, Naven Kumar et al. (2016) studied the inhibitory effect of Curcuma longa L. EO (53.10\% ar-turmerone) against $F$. graminearum and ZEA production in Sabouraud dextrose broth and in maize grains. ZEA production was completely inhibited at $3000 \mu \mathrm{g} / \mathrm{mL}$ and $3000 \mu \mathrm{g} / \mathrm{g}$, respectively. Marín et al. (2004) found that clove, lemongrass and palmarosa oils at $500 \mathrm{mg} / \mathrm{kg}$ prevented ZEA production in maize grains only at $0.950 \mathrm{a}_{\mathrm{w}} / 30^{\circ} \mathrm{C}$, while cinnamon oil had no significant effect, and oregano oil stimulated ZEA production. Considering that clove and cinnamon are high in eugenol and cinnamaldehyde, respectively, these results do not completely agree with those obtained in the present study, which may be because we have used pure compounds (IEG, $\mathrm{CINHO}$ ), different methodology and different Fusarium species. Brado Avanço et al. (2017) found that the Curcuma longa $\mathrm{L}$. EO decreased the production of $\mathrm{FB}_{1}$ and $\mathrm{FB}_{2}$ by $F$. verticillioides in liquid medium. All the concentrations of $C$. longa oil tested $(17.9-294.9 \mu \mathrm{g} / \mathrm{mL})$ caused a statistically significant decrease in fumonisin production. The $294.9 \mu \mathrm{g} / \mathrm{mL}$ concentration caused the strongest inhibition (about 90\%). A direct correlation between the inhibitory effect of fungal growth and the synthesis of $\mathrm{FB}_{1}$ and $\mathrm{FB}_{2}$ was observed. Dambolena et al. (2010) found that $O$. basilicum EO from Sagana and Kariti (different locations in Kenya) do not inhibited $\mathrm{FB}_{1}$ production by $F$. verticillioides. The first EO contained mainly LIN (more than $95 \%)$, while the second EO contained geranial (49.6\%) and neral (30.9\%) as the main constituents. However, 0 . gratissimum EOs were found to induce a significant inhibitory effect on $\mathrm{FB}_{1}$ production with respect to control $(p<0.05)$. They suggest that the high activity of the oil from 0 . gratissimum can be attributed to their high eugenol content (95.5-70.1\%), which agrees with our results. EVOH-IEG was better inhibitor of $\mathrm{FB}_{1}$ and $\mathrm{FB}_{2}$ production by $F$. proliferatum than EVOH-LIN, although the differences were not significant (Table 4).

In the food industry, EOs could also be directly mixed into the initial food formulations. This method may result in excessive amount of EO and produce changes in the taste and flavor of the food. Direct surface application of EOs, such as fumigation, may result in a rapid evaporation of active agents. Thus, in the last years many antimicrobials are incorporated at packaging material, particularly films (Nguyen Van Long et al., 2016; Zhu et al., 2016). Usually, EOs have proved to be more efficient in the vapor phase than with direct application (AguilarGonzález et al., 2015; Reyes-Jurado et al., 2019). Application of films containing EOs allows the active principle to migrate to the coating surface and provides a continuous antimicrobial effect on the food during extended exposure. Use of polymers as carriers of antimicrobials not only permits controlled release of these antimicrobials but also prevents dramatic reductions in their antimicrobial activities due to their affinity for food particles and inactivation by components in foods. It also reduces the amount of active agent required, satisfying consumer demand for fewer additives. Antimicrobial film materials must contact the surface of the food if active principle is non-volatile. EOs and their components are gaining popularity due to their 
volatile nature, which facilitates the use of small concentrations that are safe for consumption (Sivakumara and Bautista-Baños, 2014) and sensory properties of food are less affected. Paris et al. (2020) provide recent outcomes on the antimicrobial effectiveness of EOs in the vapor phase. We have also assayed EVOH films containing EOCs as a possible strategy to control the growth of toxigenic Aspergillus spp. and in the partial or total inhibition of aflatoxins and OTA biosynthesis (Mateo et al., 2017a; Tarazona et al., 2018). However, its effectiveness to control the growth of Fusarium spp., including F. culmorum and F. proliferatum, and production of their associated mycotoxins is reported for the first time in the present study.

Regarding the predictive models developed in the present study, the best overall performance in terms of average RMSE and $\mathrm{R}^{2}$ for the training datasets and for test on unseen datasets was given by RF models while the MLR models provided the worst performance. These parameters were generally better for training than for testing, as expected (Table 5, Table 6). Regarding the test set, $\mathrm{XGBoost}$ models provided similar performance to RF for predicting $\mathrm{FB}_{2}$ and outperformed NN for predicting ZEA production while NN models ranked second for the prediction of $\mathrm{FB}_{1}$ concentration.

There are very few reports concerning ML applications in the field of predictive mycology. Therefore, a comparative critical analysis of their application to predict fungal growth and mycotoxin production is difficult. Within NN methodology, Multi-layer perceptron (MLP) and radial-basis function networks (RBFN) were applied to build up a model of the joint effect of $\mathrm{a}_{\mathrm{w}}, \mathrm{pH}$ and temperature to predict the maximum specific GR of the fungus Monascus ruber. NN architectures provided better predictions for the maximum specific GR of the fungus than classical polynomial models in relation to three input variables. This agrees with our study although the input variables are not the same and the fungal species is different. The ranges of the $\mathrm{R}^{2}$ values for the RBFN, the MLP NN, and the polynomial model indicated better fit of the experimental data to the RBFN-based approach (Panagou et al., 2007; Panagou and Kodogiannis, 2009). A RBFN was developed and compared against a quadratic response surface model for predicting the specific GR of the basidiomycetous fungi Physisporinus vitreus and Neolentinus lepideus; even when both models gave reasonably good predictions, the performance of the RBFN was superior to that of the classical statistical method (Schubert et al., 2010). Mateo et al. (2009) compared RBFN and MLP with 1-2 hidden layers to predict OTA accumulation in grape juice cultures of Aspergillus carbonarius over a range of $\mathrm{a}_{\mathrm{w}}$, temperature and doses of the fungicide carbendazim. They found that training was faster for RBFN than for two-hidden-layer perceptrons, but the overall performance was worse. These ML approaches were also used to predict deoxynivalenol production in cultures of $F$. culmorum in barley seeds under different conditions of temperature, $a_{w}$, inoculum size, and incubation time. Singlehidden-layer perceptrons with a low number of hidden nodes proved better than two-hiddenlayer perceptrons, but the performance depended on the training algorithm. The RBFN reached lower errors and better generalization than MLP but they required a high number of hidden nodes (Mateo et al., 2011a). Tarazona et al. (2020b) performed a comparative study of $\mathrm{NN}, \mathrm{RF}$ and $\mathrm{XGBoost}$ models to predict the growth of $F$. culmorum and $F$. proliferatum and production of ZEA and FUM in treatments with different formulations of three chemical fungicides. In that case, XGBoost models provided slightly better results. Fast NNs were used to model the antimicrobial activity of $49 \mathrm{EOs}$ and the output data reflected the activity of these EOs against four common pathogens (Staphylococcus aureus, Escherichia coli, Candida albicans, and Clostridium perfringens) as measured by standardized disk diffusion assays; NNs were able to predict $>70 \%$ of the antimicrobial activities (Daynac et al., 2015). NNs with genetic algorithm models and surface response models were found operative and reliable in 
optimizing the minimum effective dosage of tested EOs for Candida spp. Such models reduce the number of experiments and shorten the time needed for determining the optimal antifungal doses of EOs (Perić et al., 2019). RF and gradient boosting machine (GBM) predictive models were applied to describe and predict prevalence of Listeria spp. in feces and soil samples based on meteorological factors at the farming location. Both models performed very well, similarly for the feces models, but the soil GBM model outperformed the soil RF model. According to the authors, they can be used in pastured poultry farms environments (Golden et al., 2019).

\section{Conclusions}

Our results show F. culmorum and F. proliferatum grew more rapidly at $28{ }^{\circ} \mathrm{C}$ than at $20^{\circ} \mathrm{C}$ and at $0.99 \mathrm{a}_{\mathrm{w}}$ than at $0.96 \mathrm{a}_{\mathrm{w}}$. Under the same set of conditions, the GR of $F$. culmorum was significantly higher than the GR of $F$. proliferatum. Fungal growth and ZEA and FUM production depended on $\mathrm{a}_{\mathrm{w}}$, temperature, and treatment (type of EVOH-EOC film and EOC dose). Considering the efficacy of the EVOH-EOC films assayed, as reflected by the ED values, inhibition of fungal GR, blockage of mycotoxin biosynthesis, cost and availability of the EOC, EVOH-CIT followed by EVOH-IEG and EVOH-CINHO are proposed as the most promising candidate for treatments against $F$. culmorum and F. proliferatum and as inhibitors of ZEA and FUM production. At present, implementation of lethal EOC doses against toxigenic Fusarium spp. in food, as a single effective measure to prevent fungal development and mycotoxin production, may become unreliable. However, knowledge of $E D_{50}, E D_{90}$ and $E D_{100}$ of EOCs under standardized conditions can be very useful in food technology (longer expiration periods, reduction of other chemical preservatives or application of complementary treatments, etc.). The few studies carried out to date in which ML methods have been applied to predict growth of toxigenic fungi and mycotoxin production suggest that these methods can be extremely useful in the prevention and proper management of these risks. In the present study, among the ML models studied, RF models were able to predict GR of $F$. culmorum and $F$. proliferatum, and mycotoxin (ZEA and FUM) production better than NN, XGBoost and MLR models.

\section{Declaration of competing interest}

The authors declare that they have no known competing financial interests or personal relationships that could have appeared to influence the work reported in this paper.

\section{Acknowledgments}

The authors acknowledge financial support from the European Regional Development Fund (ERDF) and Ministry of Economy and Competitiveness (MINECO, Spanish Government) (Project AGL2014-53928-C2-1-R) and ERDF and Ministry of Science, Innovation and Universities (Spanish Government) (Project RTI2018-097593-B-C22). J.V. Gómez is grateful to MINECO for a PhD contract (Ref. 543071242).

\section{References}

Aguilar-González et al., 2015. A.E. Aguilar-González, E. Palou, A. López-MaloAntifungal activity of essential oils of clove (Syzygium aromaticum) and/or mustard (Brassica nigra) in vapor phase against gray mold (Botrytis cinerea) in strawberries. Innov. Food Sci. Emerg. Technol., 32 (2015), pp. 181-185 
Bhat et al., 2010. R. Bhat, R.V. Rai, A.A. Karim. Mycotoxins in food and feed: present status and future concerns. Compr. Rev. Food Sci. Food Saf., 9 (2010), pp. 57-81

Brado Avanço et al., 2017. G. Brado Avanço, F. Dias Ferreira, N. Silva Bomfim, P.A. de Souza Rodrigues dos Santos, R. Marina Peralta, T. Brugnari, C.A. Mallman, B. Alves de Abreu Filho, J.M. Graton Mikcha, M. Machinsky Jr. Curcuma longa L. essential oil composition, antioxidant effect, and effect on Fusarium verticillioides and fumonisin production. Food Control, 73 (2017), pp. 806-813

Breiman, 2001. L. Breiman. Random forests. Mach. Learn., 45 (2001), pp. 5-32

Catalá et al., 2016. R. Catalá, V. Muriel-Galet, J.P. Cerisuelo, I. Domínguez, G. Carballo, P. Hernández-Muñoz, R. Gavara. Antimicrobial active packaging systems based on EVOH copolymers. J. Barros-Velázquez (Ed.), Antimicrobial food packaging. Chapter 22, Elsevier (2016), pp. 297-303

Cendoya et al., 2018. E. Cendoya, M.L. Chiotta, V. Zachetti, S.M. Chulze, M.L. Ramirez. Fumonisins and fumonisin-producing Fusarium occurrence in wheat and wheat by products: a review. J. Cereal Sci., 80 (2018), pp. 158-166

Cerisuelo et al., 2012. J.P. Cerisuelo, V. Muriel-Galet, J.M. Bermúdez, S. Aucejo, R. Català, R. Gavara, P. Hernández-Muñoz. Mathematical model to describe the release of an antimicrobial agent from an active package constituted by carvacrol in a hydrophilic EVOH coating on a PP film. J. Food Eng., 110 (2012), pp. 26-37

Chang et al., 2015. K.F. Chang, S.F. Hwang, R.L. Conner, H.U. Ahmed, Q. Zhou, G.D. Turnbull, S.E. Strelkov, D.L. McLaren, B.D. Gossen. First report of Fusarium proliferatum causing root rot in soybean (Glycine max L.) in Canada. Crop Prot., 67 (2015), pp. 52-58

Chen and Guestrin, 2016. T. Chen, C. Guestrin. XGBoost: a scalable tree boosting system. Proceedings of the 22nd ACM SIGKDD International Conference on Knowledge Discovery and Data Mining, August 13-17, San Francisco, CA, USA (2016), pp. 785-794,

da Cruz Cabral et al., 2013. L. da Cruz Cabral, V. Fernández-Pinto, A. Patriarca. Application of plant derived compounds to control fungal spoilage and mycotoxin production in foods. Int. J. Food Microbiol., 166 (2013), pp. 1-14

Dambolena et al., 2010. J.S. Dambolena, M.P. Zunino, A.G. López, H.R. Rubinstein, J.A. Zygadlo, J.W. Mwangi, G.N. Thoithi, I.O. Kibwage, J.M. Mwalukumbi, S.T. Kariuki. Essential oils composition of Ocimum basilicum L. and Ocimum gratissimum L. from Kenya and their inhibitory effects on growth and fumonisin production by Fusarium verticillioides. Innov. Food Sci. Emerg. Technol., 11 (2010), pp. 410-414

Daynac et al., 2015. M. Daynac, A. Cortes-Cabrera, J.M. Prieto. Application of artificial intelligence to the prediction of the antimicrobial activity of essential oils. Evid. Based Complement. Alternat. Med. (2015), Article 561024, 10.1155/2015/561024

Feijó Corrêa et al., 2018. J.A. Feijó Corrêa, P.B. Orso, K. Bordin, R.V. Hara, F.B. LucianoToxicological effects of fumonisin B1 in combination with other Fusarium toxins. Food Chem. Toxicol., 121 (2018), pp. 483-494

Gao et al., 2016. M.L. Gao, Y.S. Luan, H.N. Yu, Y.M. Bao. First report of tomato leaf spot caused by Fusarium proliferatum in China. Can. J. Plant Pathol., 38 (2016), pp. 400-404 
Gil-Serna et al., 2013. J. Gil-Serna, E.M. Mateo, M.T. González-Jaén, M. Jiménez, C. Vázquez, B. Patiño. Contamination of barley seeds with Fusarium species and their toxins in Spain: an integrated approach. Food Addit. Contam. Part A, 30 (2013), pp. 372-380

Golden et al., 2019. C.E. Golden, M.J. Rothrock, A. Mishra. Comparison between random forest and gradient boosting machine methods for predicting Listeria spp. prevalence in the environment of pastured poultry farms. Food Res. Int., 122 (2019), pp. 47-55

Gómez et al., 2018. J.V. Gómez, A. Tarazona, R. Mateo-Castro, J.V. Gimeno-Adelantado, M. Jiménez, E.M. Mateo. Selected plant essential oils and their main active components, a promising approach to inhibit aflatoxigenic fungi and aflatoxin production in food. Food Addit. Contam. Part A, 35 (2018), pp. 1581-1595

Günther and Fritsch, 2010. F. Günther, S. Fritsch. Neuralnet: training of neural networks. R J., 2 (1) (2010), pp. 30-38

Hashem et al., 2010. M. Hashem, A.M. Moharama, A.A. Zaied, F.E.M. Saleh. Efficacy of essential oils in the control of cumin root rot disease caused by Fusarium spp. Crop Prot., 29 (2010), pp. 1111-1117

Kuhn, 2016. M. Kuhn. Contributions from Wing, J., Weston, S., Williams, A., Keefer, Ch., Engelhardt, A., Cooper, T., Mayer, Z., Kenkel, B., the R Core Team, Benesty, M., Lescarbeau, R., Ziem, A., Scrucca, L., Tang, Y., Candan, C. Caret: classification and regression training. $\mathbf{R}$ package version 6.0-71. https://CRAN.R-project.org/package=caret (2016)

Laraba et al., 2017. I. Laraba, H. Boureghda, N. Abdallah, O. Bouaicha, F. Obanor, A. Moretti, D.M. Geiser, H.S. Kim, S.P. McCormick, R.H. Proctor, A.C. Kelly, T.J. Ward, K. O'Donnell. Population genetic structure and mycotoxin potential of the wheat crown rot and head blight pathogen Fusarium culmorum in Algeria. Fungal Genet. Biol. (2017), pp. 34-41

Liaw and Wiener, 2002. A. Liaw, M.C. Wiener. Classification and regression by randomForest R News, 2 (3) (2002), pp. 18-22.

Magan et al., 2002. N. Magan, R. Hope, A. Colleate, E.S. Baxter. Relationship between growth and mycotoxin production by Fusarium species, biocides and environment. Eur. J. Plant Pathol., 108 (2002), pp. 685-690

Magan et al., 2003. N. Magan, R. Hope, V. Cairns, D. AldredPost-harvest fungal ecology: impact of fungal growth and mycotoxin accumulation in stored grain. Eur. J. Plant Pathol., 109 (2003), pp. 723-730

Manganyi et al., 2015.M.C. Manganyi, T. Regnier, E.I. Olivier. Antimicrobial activities of selected essential oils against Fusarium oxysporum isolates and their biofilms. S. Afr. J. Bot., 99 (2015), pp. 115-121

Marín et al., 2004. S. Marín, A. Velluti, A.J. Ramos, V. Sanchis. Effect of essential oils on zearalenone and deoxynivalenol production by Fusarium graminearum in non-sterilized maize grain. Food Microbiol., 21 (2004), pp. 313-318

Marín et al., 2013. P. Marín, A. de Ory, A. Cruz, N. Magan, M.T. González-Jaén. otential effects of environmental conditions on the efficiency of the antifungal tebuconazole controlling Fusarium verticillioides and Fusarium proliferatum growth rate and fumonisin biosynthesis. Int. J. Food Microbiol., 165 (2013), pp. 251-258 
Mateo et al., 2009. F. Mateo, R. Gadea, A. Medina, R. Mateo, M. Jiménez. Predictive assessment of ochratoxin $A$ accumulation in grape juice based-medium by Aspergillus carbonarius using neural networks. J. Appl. Microbiol., 107 (2009), pp. 915-927

Mateo et al., 2011a. F. Mateo, R. Gadea, E.M. Mateo, M. Jiménez. Multilayer perceptron neural networks and radial-basis function networks as tools to forecast accumulation of deoxynivalenol in barley seeds contaminated with Fusarium culmorum. Food Control, 22 (2011), pp. 88-95

Mateo et al., 2011b. E.M. Mateo, F.M. Valle-Algarra, R. Mateo-Castro, M. Jiménez, N. Magan. Effect of fenpropimorph, prochloraz and tebuconazole on growth and production of T-2 and HT-2 toxins by Fusarium langsethiae in oat-based medium. Int. J. Food Microbiol., 151 (2011), pp. 289-298

Mateo et al., 2013. E.M. Mateo, F.M. Valle-Algarra, M. Jiménez, N. Magan. Impact of three sterol-biosynthesis inhibitors on growth of Fusarium langsethiae and on T-2 and HT-2 toxin production in oat grain under different ecological conditions. Food Control, 34 (2013), pp. 521-529

Mateo et al., 2017a. E.M. Mateo, J.V. Gómez, I. Domínguez, J.V. Gimeno-Adelantado, R. Mateo-Castro, R. Gavara, M. Jiménez. Impact of bioactive packaging systems based on EVOH films and essential oils in the control of aflatoxigenic fungi and aflatoxin production in maize. Int. J. Food Microbiol., 254 (2017), pp. 36-46

Mateo et al., 2017b. E.M. Mateo, J.V. Gómez, J.V. Gimeno-Adelantado, D. Romera, R. MateoCastro, M. Jiménez. Assessment of azole fungicides as a tool to control growth of Aspergillus flavus and aflatoxin B1 and B2 production in maize. Food Addit. Contam. Part A, 34 (2017), pp. 1039-1051

Meher et al., 2019. P.K. Meher, T.K. Sahu, S. Gahoi, R. Tomar, A.R. Rao. FunbarRF: DNA barcode-based fungal species prediction using multiclass Random Forest supervised learning model. BMC Genet., 20 (2019), p. 2, 10.1186/s12863-018-0710-z2

Muriel-Galet et al., 2012. V. Muriel-Galet, G. López-Carballo, R. Gavara, P. Hernández-Muñoz. Antimicrobial food packaging film based on the release of LAE from EVOH. Int. J. Food Microbiol., 157 (2012), pp. 39-244

Muriel-Galet et al., 2013. V. Muriel-Galet, J.P. Cerisuelo, G. López-Carballo, S. Aucejo, R. Gavara, P. Hernández-Muñoz. Evaluation of EVOH-coated PP films with oregano essential oil and citral to improve the shelf-life of packaged salad. Food Control, 30 (2013), pp. 137-143

Naven Kumar et al., 2016. K. Naven Kumar, M. Venkataramana, J.A. Allen, S. Chandranayaka, H.S. Murali, H.V. Batra. Role of Curcuma longa L. essential oil in controlling the growth and zearalenone production of Fusarium graminearum. LWT Food Sci. Technol., 69 (2016), pp. 522-528, 10.1016/j.Iwt.2016.02.005

.Nguyen Van Long et al., 2016. N. Nguyen Van Long, C. Joly, P. DantignyActive packaging with antifungal activities. Int. J. Food Microbiol., 220 (2016), pp. 73-90

Ostry et al., 2017. V. Ostry, F. Malir, J. Toman, Y. Grosse. Mycotoxins as human carcinogens the IARC monographs classification. Mycotox. Res., 33 (2017), pp. 65-73 
Panagou and Kodogiannis, 2009. E.Z. Panagou, V.S. Kodogiannis. Application of neural networks as a nonlinear modelling technique in food mycology. Expert Syst. Appl., 36 (2009), pp. 121-131

Panagou et al., 2007. E.Z. Panagou, V. Kodogiannis, G.J.E. NychasModelling fungal growth using radial basis function neural networks: the case of the ascomycetous fungal Monascus ruber van Tiegham. Int. J. Food Microbiol., 117 (2007), pp. 276-286

Paris et al., 2020. M.J. Paris, N. Ramírez-Corona, E. Palou, A. López-Malo. Modelling release mechanisms of cinnamon (Cinnamomum zeylanicum) essential oil encapsulated in alginate beads during vapor-phase application. J. Food Eng., 282 (2020), p. 110024, 10.1016/j.jfoodeng.2020.110024

Perić et al., 2019. M. Perić, K. Rajković, A. Milić Lemić, R. Živković, V. Arsić Arsenijević. Development and validation of mathematical models for testing antifungal activity of different essential oils against Candida species. Arch. Oral Biol., 98 (2019), pp. 258-264

Prakash et al., 2015. B. Prakash, A. Kedia, P.K. Mishra, N.K. DubeyPlant essential oils as food preservatives to control moulds, mycotoxin contamination and oxidative deterioration of agri-food commodities-potentials and challenges. Food Control, 47 (2015), pp. 381-391

Qu et al., 2019. K. Qu, G. Fai, L. Xiangrong, L. Yuan, Z. QuanApplication of machine learning in microbiology. Front. Microbiol., 10 (2019), p. 10, 10.3389/fmicb.2019.00827art.287

Reyes-Jurado et al., 2019. F. Reyes-Jurado, T. Cervantes-Rincón, H. Bach, A. López-Malo, E. Palou. Antimicrobial activity of Mexican oregano (Lippia berlandieri), thyme (Thymus vulgaris), and mustard (Brassica nigra) essential oils in gaseous phase. Ind. Crop. Prod., 131 (2019), pp. 90-95

Ribeiro-Santos et al., 2017. R. Ribeiro-Santos, M. Andrade, N. Ramos de Melo, A. Sanches-Silva. Use of essential oils in active food packaging: recent advances and future trends. Trends Food Sci. Technol., 61 (2017), pp. 132-140

Romera et al., 2018. D. Romera, E.M. Mateo, R. Mateo-Castro, J.V. Gómez, J.V. GimenoAdelantado, M. Jiménez. Determination of multiple mycotoxins in feedstuffs by combined use of UPLC-MS/MS and UPLC-QTOF-MS. Food Chem., 267 (2018), pp. 140-148

Saleh et al., 2017. A.A. Saleh, A.H. Sharafaddin, M.H. El-Komy, Y.E. Ibrahim, Y.K. Hamad, Y.Y. Molan. Fusarium species associated with date palm in Saudi Arabia. Eur. J. Plant Pathol., 148 (2017), pp. 367-377

Schubert et al., 2010. M. Schubert, S. Mourad, F. Schwarze. Radial basis function neural networks for modeling growth rates of the basidiomycetes Physisporinus vitreus and Neolentinus lepideus. Appl. Microbiol. Biotechnol., 85 (2010), pp. 703-712

Seseni et al., 2015. L. Seseni, T. Regnier, M.P. Roux-van der Merwe, E. Mogale, J. Badenhorst. Control of Fusarium spp. causing damping-off of pine seedlings by means of selected essential oils. Ind. Crop. Prod., 76 (2015), pp. 329-332

Sharma et al., 2017. A. Sharma, S. Rajendran, A. Srivastava, S. Sharma, B. Kundu. Antifungal activities of selected essential oils against Fusarium oxysporum f. sp. lycopersici 1322, with emphasis on Syzygium aromaticum essential oil. J. Biosci. Bioeng., 123 (2017), pp. 308-313 
Sivakumara and Bautista-Baños, 2014. D. Sivakumara, S. Bautista-BañosA review on the use of essential oils for postharvest decay control and maintenance of fruit quality during storage. Crop Prot., 64 (2014), pp. 27-37

Stepien et al., 2011. L. Stepien, G. Koczyk, A. WaskiewiczGenetic and phenotypic variation of Fusarium proliferatum isolates from different host species. J. Appl. Genet., 52 (2011), pp. 487-496

Suppakul et al., 2003. P. Suppakul, J. Miltz, K. Sonneveld, S.W. BiggerActive packaging technologies with an emphasis on antimicrobial packaging and its applications. J. Food Sci., 68 (2003), pp. 408-420

Tarazona et al., 2018. A. Tarazona, J.V. Gómez, R. Gavara, R. Mateo-Castro, J.V. GimenoAdelantado, M. Jiménez, E.M. Mateo. Risk management of ochratoxigenic fungi and ochratoxin A in maize grains by bioactive EVOH films containing individual components of some essential oils. Int. J. Food Microbiol., 269 (2018), pp. 107-119

Tarazona et al., 2020a. A. Tarazona, J.V. Gómez, M. Jiménez, F. Mateo, D. Romera, E.M. Mateo. Study on mycotoxin contamination of maize kernels in Spain. Food Control, 118 (2020), p. 107370, 10.1016/j.foodcont.2020.107370

Tarazona et al., 2020b. A. Tarazona, E.M. Mateo, J.V. Gómez, D. Romera, F. Mateo. Potential use of machine learning methods in assessment of Fusarium culmorum and F. proliferatum growth and mycotoxin production in treatments with antifungal agents. Fungal Biol. (2020), 10.1016/j.funbio.2019.11.006

Vilaplana et al., 2018. R. Vilaplana, K. Pérez-Revelo, S. Valencia-ChamorroEssential oils as an alternative postharvest treatment to control fusariosis, caused by Fusarium verticillioides, in fresh pineapples (Ananas comosus). Sci. Hortic., 238 (2018), pp. 255-263

Wan et al., 2020. J. Wan, Z. Jin, S. Zhong, P. Schwarz, B. Chen, J. Rao. Clove oil-in-water nanoemulsion: mitigates growth of Fusarium graminearum and trichothecene mycotoxin production during the malting of Fusarium infected barley. Food Chem., 312 (2020), p. 126120

Waśkiewicz et al., 2012. A. Waśkiewicz, M. Beszterda, P. GolińskiReview. Occurrence of fumonisins in food - an interdisciplinary approach to the problem. Food Control, 26 (2012), pp. 491-499

Wegulo et al., 2015. S.N. Wegulo, P.S. Baenziger, J. Hernandez Nopsa, W.W. Bockus, H. HallenAdamsManagement of Fusarium head blight of wheat and barley. Crop Prot., 73 (2015), pp. 100-107

Xing et al., 2014. F. Xing, H. Hua, J.N. Selvaraj, Y. Zhao, L. Zhou, X. Liu, Y. Liu. Growth inhibition and morphological alterations of Fusarium verticillioides by cinnamon oil and cinnamaldehyde. Food Control, 46 (2014), pp. 343-350

Yang et al., 2018, D. Yang, X. Jiang, J. Sun, X. Li, X. Li, R. Jiao, Z. Peng, Y. Li, W. Bay. Toxic effects of zearalenone on gametogenesis and embryonic development: a molecular point of review. Food Chem. Toxicol., 119 (2018), pp. 24-30 
Zhang et al., 2015. H. Zhang, M. Hortal, A. Dobon, J.M. Bermúdez, M.L. Lledo. The effect of active packaging on minimizing food losses: life cycle assessment (LCA) of essential oil component-enabled. Packaging for fresh beef. Packag. Technol. Sci., 28 (2015), pp. 761-774

Zhu et al., 2016. Y. Zhu, Y.I. Hassan, C. Watts, T. Zhou. Innovative technologies for the mitigation of mycotoxins in animal feed and ingredients-a review of recent patents. Anim. Feed Sci. Technol., 216 (2016), pp. 19-29, 10.1016/j.anifeedsci.2016.03.030 
Table 1. Main parameters for each machine learning (ML) model and their tuning values.

\begin{tabular}{|c|c|c|c|}
\hline ML model & Parameter name & Description & Values \\
\hline \multirow[t]{2}{*}{ Neural network } & size & Hidden layer units (nodes) & {$[5,10,15,20]$} \\
\hline & decay & Weight decay rate & {$[0.1,0.2,0.3]$} \\
\hline \multirow[t]{2}{*}{ Random forest } & mtry & $\begin{array}{l}\text { Number of randomly } \\
\text { selected predictors }\end{array}$ & {$[2,3,4]$} \\
\hline & ntree & Number of trees & 500 \\
\hline \multirow{7}{*}{$\begin{array}{l}\text { Extreme gradient } \\
\text { boosted tree }\end{array}$} & nrounds & Number of iterations & 150 \\
\hline & max_depth & Maximum tree depth & {$[1,2,3,4,5]$} \\
\hline & eta & Learning rate or shrinkage & {$[0.1,0.2,0.3]$} \\
\hline & gamma & $\begin{array}{l}\text { Regularization penalty } \\
\text { factor }\end{array}$ & 0 \\
\hline & colsample_bytree & $\begin{array}{l}\text { Fraction of columns to be } \\
\text { randomly sampled for each } \\
\text { tree }\end{array}$ & 1 \\
\hline & min_child_weight & $\begin{array}{l}\text { Minimum instance weight } \\
\text { per node }\end{array}$ & 0.5 \\
\hline & subsample & $\begin{array}{l}\text { Subsample ratio from } \\
\text { training set to grow the } \\
\text { trees }\end{array}$ & {$[0.5,0.75,1]$} \\
\hline
\end{tabular}


Table 2. Arrangement of homogeneous groups by post hoc Tukey's HSD test $(\alpha=0.05)$ within EVOH-EOC film type, EOC dose, temperature and water activity with regard to their effects on F. culmorum and $F$. proliferatum growth rate on partly milled maize kernels.

\begin{tabular}{|c|c|c|c|}
\hline \multirow[t]{4}{*}{ Factor } & Level & \multicolumn{2}{|l|}{ Fungal species } \\
\hline & & F. culmorum & F. proliferatum \\
\hline & & Growth rate & Growth rate \\
\hline & & Low $\rightarrow$ high & Low $\rightarrow$ high \\
\hline \multirow[t]{4}{*}{ EVOH-EOC film type } & CIT & \multirow{4}{*}{ • } & \multirow[t]{4}{*}{$\bullet$} \\
\hline & $\mathrm{CINHO}$ & & \\
\hline & IEG & & \\
\hline & LIN & & \\
\hline \multirow{4}{*}{$\begin{array}{l}\text { Dose ( } \mu \mathrm{g} \text { EOC/Petri } \\
\text { dish) }\end{array}$} & 333 & \multirow{4}{*}{$\begin{array}{lll} & \bullet \\
\bullet & \quad \\
\bullet & \quad\end{array}$} & \multirow{4}{*}{$\begin{array}{ll} & \bullet \\
\bullet & \end{array}$} \\
\hline & 666 & & \\
\hline & 1665 & & \\
\hline & 3330 & & \\
\hline \multirow[t]{2}{*}{ Temperature $\left({ }^{\circ} \mathrm{C}\right)$} & 20 & \multirow[t]{2}{*}{$\bullet$} & \multirow[t]{2}{*}{$\bullet$} \\
\hline & 28 & & \\
\hline \multirow[t]{2}{*}{ Water activity $\left(a_{w}\right)$} & 0.96 & \multirow{2}{*}{$\begin{array}{l} \\
\bullet\end{array}$} & \multirow[t]{2}{*}{$\bullet$} \\
\hline & 0.99 & & \\
\hline
\end{tabular}

Within each column, the levels containing a black circle form a group of means within which the Tukey's HSD test does not find statistically significant differences $(\alpha=0.05)$. Black circles in the same row denote overlapping. 
Table 3. Effective doses $\left(E D_{50}, E D_{90}\right.$ and $\left.E D_{100}\right)$ in vapor phase of $E V O H$ films containing cinnamaldehyde (CINHO), citral (CIT), isoeugenol (IEG) or linalool (LIN) $(\mu \mathrm{g} /$ fungal culture in 9$\mathrm{cm}$ Petri dish) against $F$. culmorum and $F$. proliferatum on partly milled maize under different environmental conditions.

\begin{tabular}{|c|c|c|c|c|c|c|c|c|c|c|c|c|c|c|}
\hline \multirow[t]{2}{*}{ Fungi } & \multirow[t]{2}{*}{$\begin{array}{l}\mathrm{T} \\
\left({ }^{\circ} \mathrm{C}\right)\end{array}$} & \multirow[t]{2}{*}{$a_{w}$} & \multicolumn{3}{|c|}{ EVOH-CINHO } & \multicolumn{3}{|c|}{ EVOH-CIT } & \multicolumn{3}{|c|}{ EVOH-IEG } & \multicolumn{3}{|c|}{ EVOH-LIN } \\
\hline & & & $E_{50}$ & $\mathrm{ED}_{90}$ & $\mathrm{ED}_{100}$ & $\mathrm{ED}_{50}$ & $E D_{90}$ & $\mathrm{ED}_{100}$ & $E_{50}$ & $E_{90}$ & $\mathrm{ED}_{100}$ & $\mathrm{ED}_{50}$ & $E D_{90}$ & $\mathrm{ED}_{100}$ \\
\hline \multirow{4}{*}{ F. culmorum } & 20 & 0.96 & 2500 & $>3330$ & $>3330$ & 455 & 625 & 660 & 1100 & $>3330$ & $>3330$ & 2150 & $>3330$ & $>3330$ \\
\hline & & 0.99 & 510 & 1330 & 1660 & 200 & 450 & 660 & 240 & 1400 & 3330 & 470 & $>3330$ & $>3330$ \\
\hline & 28 & 0.96 & 570 & 2400 & 3330 & 225 & 540 & 660 & 290 & $>3330$ & $>3330$ & 640 & $>3330$ & $>3330$ \\
\hline & & 0.99 & 3080 & $>3330$ & $>3330$ & 300 & 590 & 660 & 1650 & $>3330$ & $>3330$ & 645 & $>3330$ & $>3330$ \\
\hline \multirow{4}{*}{$\begin{array}{l}\text { F. } \\
\text { proliferatum }\end{array}$} & \multirow{2}{*}{20} & 0.96 & 490 & 630 & 660 & 435 & 620 & 660 & 1920 & 3330 & $>3330$ & 1600 & $>3330$ & $>3330$ \\
\hline & & 0.99 & 495 & 630 & 660 & 490 & 630 & 660 & 1670 & $>3330$ & $>3330$ & $>3330$ & $>3330$ & $>3330$ \\
\hline & \multirow{2}{*}{28} & 0.96 & 480 & 630 & 660 & 460 & 625 & 660 & 1740 & $>3330$ & $>3330$ & $>3330$ & $>3330$ & $>3330$ \\
\hline & & 0.99 & 615 & $>3330$ & $>3330$ & 430 & 617 & 660 & 670 & $>3330$ & $>3330$ & $>3330$ & $>3330$ & $>3330$ \\
\hline
\end{tabular}


Table 4. Arrangement of homogeneous groups by post hoc Tukey's HSD test $(\alpha=0.05)$ within EVOH-EOC film type, EOC dose, temperature and water activity with regard to their effects on production of zearalenone (ZEA) by F. culmorum and production of fumonisins ( $\mathrm{FB}_{1}$ and $\mathrm{FB}_{2}$ ) by F. proliferatum on partly milled maize kernels. Incubation time: 21 days.

\begin{tabular}{|c|c|c|c|c|c|c|c|c|c|c|c|}
\hline \multirow[t]{4}{*}{ Factor } & \multirow[t]{4}{*}{ Level } & \multicolumn{10}{|c|}{ Fungal species } \\
\hline & & \multirow{2}{*}{\multicolumn{4}{|c|}{$\begin{array}{l}\text { F. culmorum } \\
\text { ZEA }\end{array}$}} & \multicolumn{6}{|c|}{ F. proliferatum } \\
\hline & & & & & & \multicolumn{3}{|c|}{ FB1 } & \multicolumn{3}{|c|}{ FB2 } \\
\hline & & \multicolumn{4}{|c|}{ Low $\rightarrow$ high } & \multicolumn{3}{|c|}{ Low $\rightarrow$ high } & \multicolumn{3}{|c|}{ Low $\rightarrow$ high } \\
\hline \multirow[t]{4}{*}{ EVOH-EOC film type } & CIT & - & & & & $\bullet$ & & & $\bullet$ & & \\
\hline & CINHO & & $\bullet$ & & & $\bullet$ & & & $\bullet$ & & \\
\hline & IEG & - & & & & & $\bullet$ & & & $\bullet$ & \\
\hline & LIN & & $\bullet$ & & & & $\bullet$ & & & $\bullet$ & \\
\hline \multirow[t]{4}{*}{ Dose ( $\mu \mathrm{g}$ EOC/Petri dish) } & 333 & & & & $\bullet$ & & $\bullet$ & & & & $\bullet$ \\
\hline & 666 & & & $\bullet$ & & $\bullet$ & & & & $\bullet$ & \\
\hline & 1665 & & $\bullet$ & & & $\bullet$ & & & & $\bullet$ & \\
\hline & 3330 & $\bullet$ & & & & $\bullet$ & & & $\bullet$ & & \\
\hline \multirow[t]{2}{*}{ Temperature $\left({ }^{\circ} \mathrm{C}\right)$} & 20 & $\bullet$ & & & & $\bullet$ & & & $\bullet$ & & \\
\hline & 28 & & $\bullet$ & & & $\bullet$ & & & $\bullet$ & & \\
\hline \multirow[t]{2}{*}{ Water activity $\left(\mathrm{a}_{\mathrm{w}}\right)$} & 0.96 & & $\bullet$ & & & & & $\bullet$ & $\bullet$ & & \\
\hline & 0.99 & & $\bullet$ & & & $\bullet$ & & & $\bullet$ & & \\
\hline
\end{tabular}

Within each column, the levels containing a black circle form a group of means within which the Tukey's HSD test does not find statistically significant differences $(\alpha=0.05)$. 
Table 5. Best machine learning (ML) model performance for predicting growth rate (GR) and ZEA production by $F$. culmorum and $\mathrm{GR}$, and $\mathrm{FB}_{1} / \mathrm{FB}_{2}$ production by $F$. proliferatum cultured on partly milled maize with $\mathrm{EVOH}$ films containing cinnamaldehyde, citral, isoeugenol or linalool.

\begin{tabular}{|c|c|c|c|c|}
\hline Output variable & ML model tested & Best model parameters & RMSE $^{a}$ & $\mathbf{R}^{2}$ \\
\hline \multirow{3}{*}{ F. culmorum GR } & $N N^{b}$ & size $=5$, decay $=0.3$ & 1.936 & 0.868 \\
\hline & $\mathrm{RF}^{\mathrm{c}}$ & ntree $=500$, mtry $=4$ & 1.803 & 0.886 \\
\hline & $X G B o o s t^{d}$ & $\begin{array}{l}\text { max_depth }=3, \text { eta }=0.1, \\
\text { subsample }=0.75\end{array}$ & 2.243 & 0.814 \\
\hline \multirow{3}{*}{$\begin{array}{l}\text { ZEA production by } F \text {. } \\
\text { culmorum }\end{array}$} & NN & size $=5$, decay $=0.3$ & 1.130 & 0.684 \\
\hline & RF & ntree $=500$, mtry $=4$ & 1.032 & 0.708 \\
\hline & XGBoost & $\begin{array}{l}\text { max_depth }=3, \text { eta }=0.1, \\
\text { subsample }=1\end{array}$ & 1.041 & 0.736 \\
\hline \multirow{3}{*}{ F. proliferatum GR } & $\mathrm{NN}$ & size $=10$, decay $=0.1$ & 0.608 & 0.940 \\
\hline & $\mathrm{RF}$ & ntree $=500$, mtry $=4$ & 0.563 & 0.948 \\
\hline & XGBoost & $\begin{array}{l}\text { max_depth }=2, \text { eta }=0.2 \\
\text { subsample }=1\end{array}$ & 0.687 & 0.965 \\
\hline \multirow{3}{*}{$\begin{array}{l}\mathrm{FB}_{1} \text { production by } F \text {. } \\
\text { proliferatum }\end{array}$} & $\mathrm{NN}$ & size $=5$, decay $=0.1$ & 0.599 & 0.886 \\
\hline & $\mathrm{RF}$ & ntree $=500$, mtry $=4$ & 0.593 & 0.914 \\
\hline & XGBoost & $\begin{array}{l}\text { max_depth }=3, \text { eta }=0.3 \\
\text { subsample }=1\end{array}$ & 0.859 & 0.824 \\
\hline \multirow{3}{*}{$\begin{array}{l}\mathrm{FB}_{2} \text { production by } F . \\
\text { proliferatum }\end{array}$} & $\mathrm{NN}$ & size $=20$, decay $=0.1$ & 0.449 & 0.900 \\
\hline & $\mathrm{RF}$ & ntree $=500$, mtry $=4$ & 0.428 & 0.916 \\
\hline & XGBoost & $\begin{array}{l}\text { max_depth }=4, \text { eta }=0.1 \\
\text { subsample }=1\end{array}$ & 0.444 & 0.894 \\
\hline
\end{tabular}

${ }^{a}$ Root mean squared error; ${ }^{b} \mathrm{NN}$ : Neural network (single-hidden-layer perceptrons); ${ }^{\mathrm{c} R F}$ : Random Forest; ${ }^{\mathrm{d}}$ XGBoost: Extreme gradient boosted trees (the following parameters had constant values: nrounds $=150$, gamma = 0 , colsample_bytree $=1$, min_child_weight $=0.5$ ). 
Table 6. Performance of the ML models (Table 5) for predicting GR and ZEA production by $F$. culmorum and $\mathrm{GR}$, and $\mathrm{FB}_{1} / \mathrm{FB}_{2}$ production by $F$. proliferatum cultured on partly milled maize with $\mathrm{EVOH}$ films containing EOC on a test set not used for model design.

\begin{tabular}{|l|l|l|l|}
\hline \multicolumn{1}{|c|}{ Output variable } & Model tested $^{-}$ & RMSE $^{\mathbf{a}}$ & \multicolumn{1}{c|}{$\mathbf{R}^{\mathbf{2}}$} \\
\hline \multirow{5}{*}{ F. culmorum GR } & $\mathrm{NN}^{\mathrm{b}}$ & 2.395 & 0.771 \\
\cline { 2 - 4 } & $\mathrm{RF}^{\mathrm{c}}$ & 2.248 & 0.840 \\
\cline { 2 - 4 } & $\mathrm{XGBoost}^{\mathrm{d}}$ & 2.708 & 0.726 \\
\cline { 2 - 4 } & $\mathrm{MLR}^{\mathrm{e}}$ & 2.914 & 0.654 \\
\hline \multirow{5}{*}{ ZEA production by F. culmorum } & $\mathrm{NN}$ & 1.283 & 0.480 \\
\cline { 2 - 4 } & $\mathrm{RF}$ & 1.031 & 0.654 \\
\cline { 2 - 4 } & $\mathrm{XGBoost}$ & 1.254 & 0.525 \\
\cline { 2 - 4 } & $\mathrm{MLR}$ & 1.324 & 0.480 \\
\hline \multirow{5}{*}{$\mathrm{FB}_{1}$ production by F. proliferatum } & $\mathrm{NN}$ & 0.721 & 0.916 \\
\cline { 2 - 4 } & $\mathrm{RF}$ & 0.618 & 0.957 \\
\cline { 2 - 4 } & $\mathrm{XGBoost}$ & 0.712 & 0.928 \\
\cline { 2 - 4 } & $\mathrm{MLR}$ & 0.929 & 0.879 \\
\hline \multirow{5}{*}{$\mathrm{FB}_{2}$ production by F. proliferatum } & $\mathrm{NN}$ & 0.822 & 0.772 \\
\cline { 2 - 4 } & $\mathrm{RF}$ & 0.861 & 0.822 \\
\cline { 2 - 4 } & $\mathrm{XGBoost}$ & 0.992 & 0.745 \\
\cline { 2 - 4 } & $\mathrm{MLR}$ & 1.484 & 0.493 \\
\hline & $\mathrm{NN}$ & 0.605 & 0.772 \\
\cline { 2 - 4 } & $\mathrm{RF}$ & 0.501 & 0.847 \\
\cline { 2 - 4 } & $\mathrm{XGBoost}$ & 0.503 & 0.857 \\
\cline { 2 - 4 } & $\mathrm{MLR}$ & 1.426 & 0.617 \\
\hline
\end{tabular}

${ }^{a}$ Root mean squared error for test. ${ }^{b} \mathrm{NN}$ : Neural network (single-hidden-layer perceptron). ${ }^{\mathrm{c}}$ RF: Random Forest. ${ }^{d}$ XGBoost: Extreme Gradient Boosted trees. ${ }^{e}$ Multiple linear regression. 

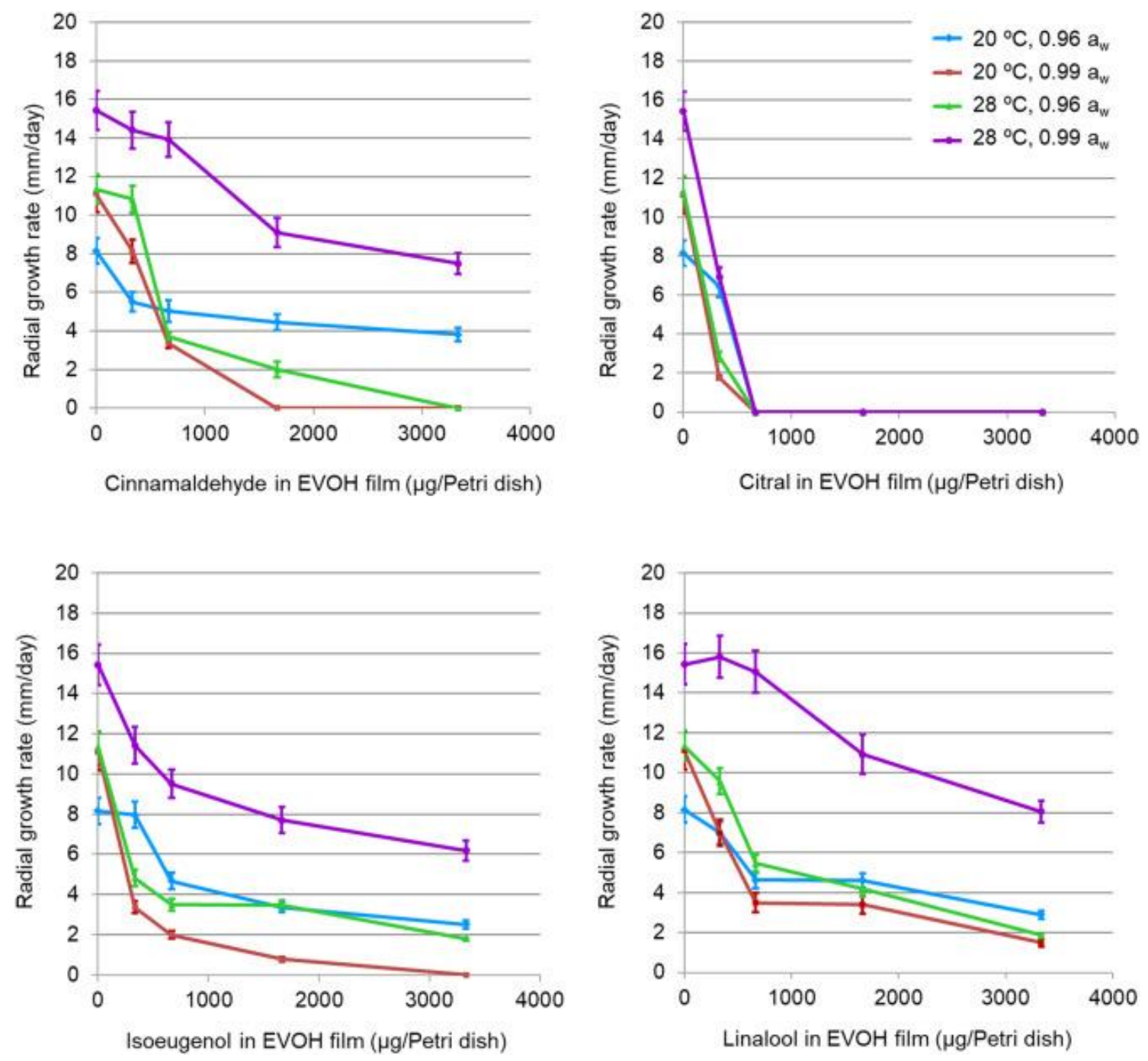

Fig. 1. Radial growth rates $(G R)$ of $F$. culmorum in cultures on partially milled maize grain incubated with $\mathrm{EVOH}$ films containing cinnamaldehyde, citral, isoeugenol or linalool at different doses in vapor phase under different $\mathrm{a}_{\mathrm{w}}$ and temperature regimes. Incubation period: 21 days. Error bars represent standard deviations. 

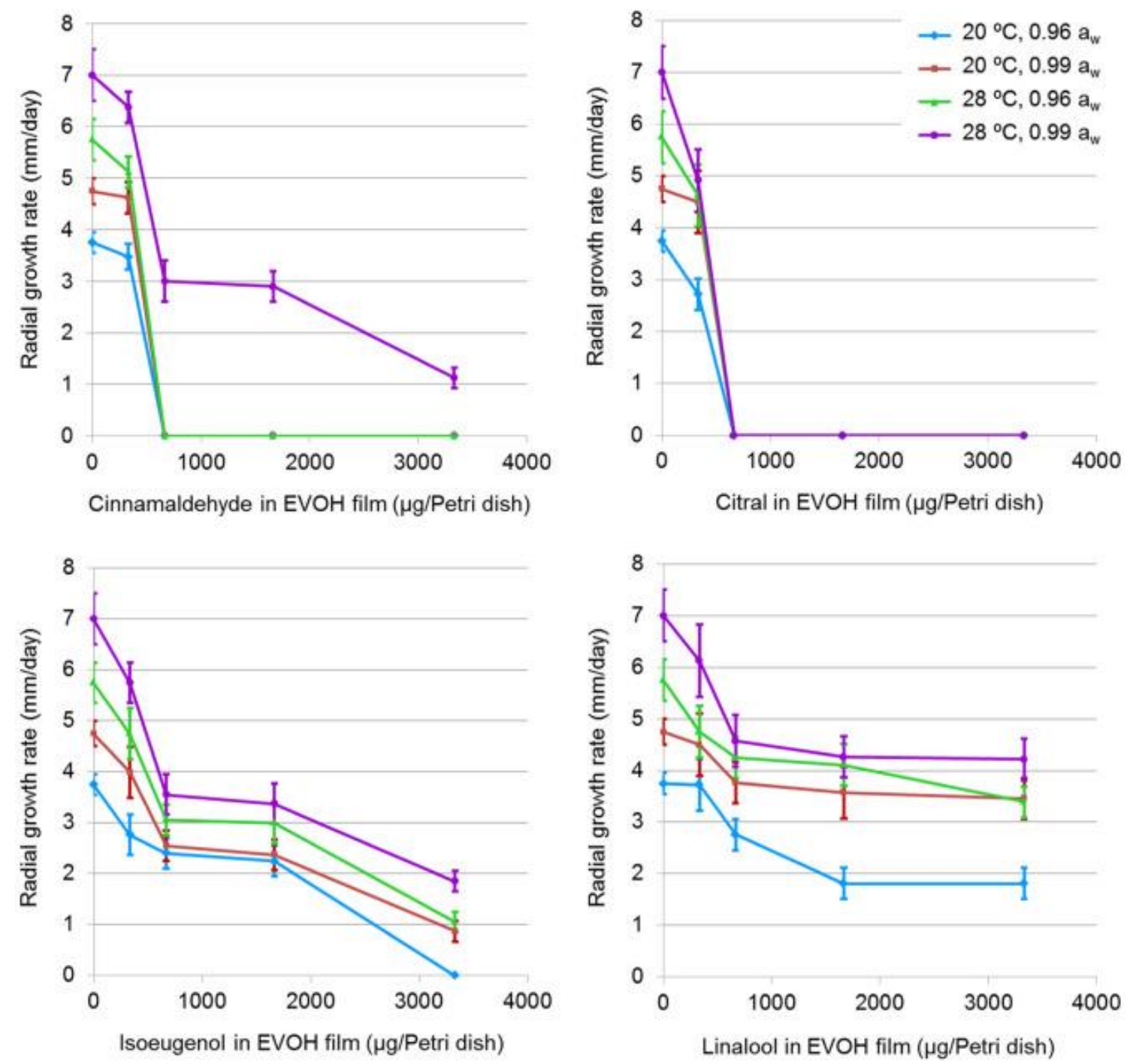

Fig. 2. Radial growth rates (GR) of $F$. proliferatum in cultures on partially milled maize grain incubated with $\mathrm{EVOH}$ films containing cinnamaldehyde, citral, isoeugenol or linalool at different doses in vapor phase under different $a_{w}$ and temperature regimes. Incubation period: 21 days. Error bars represent standard deviations. 


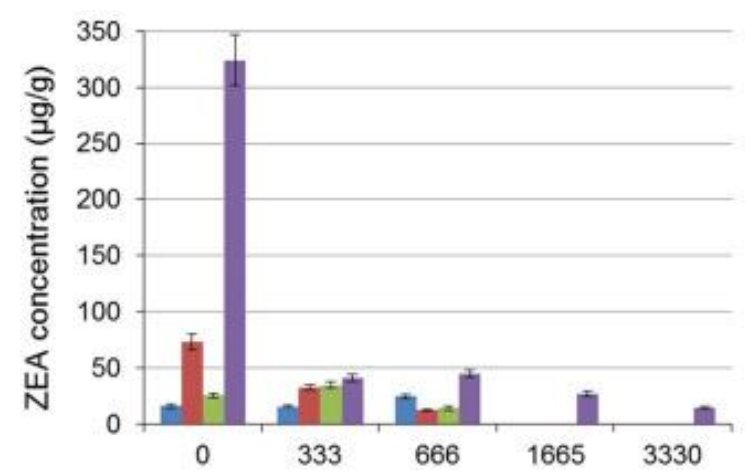

Cinnamaldehyde in EVOH film ( $\mu \mathrm{g} /$ Petri dish)

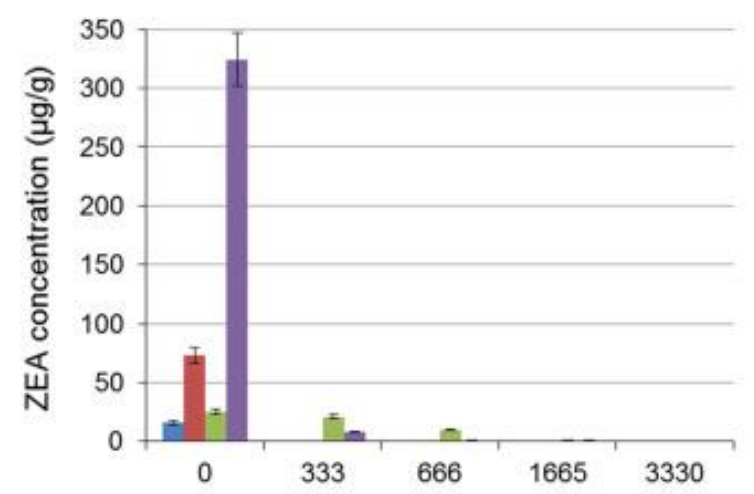

Isoeugenol in EVOH film ( $\mu \mathrm{g} /$ Petri dish)
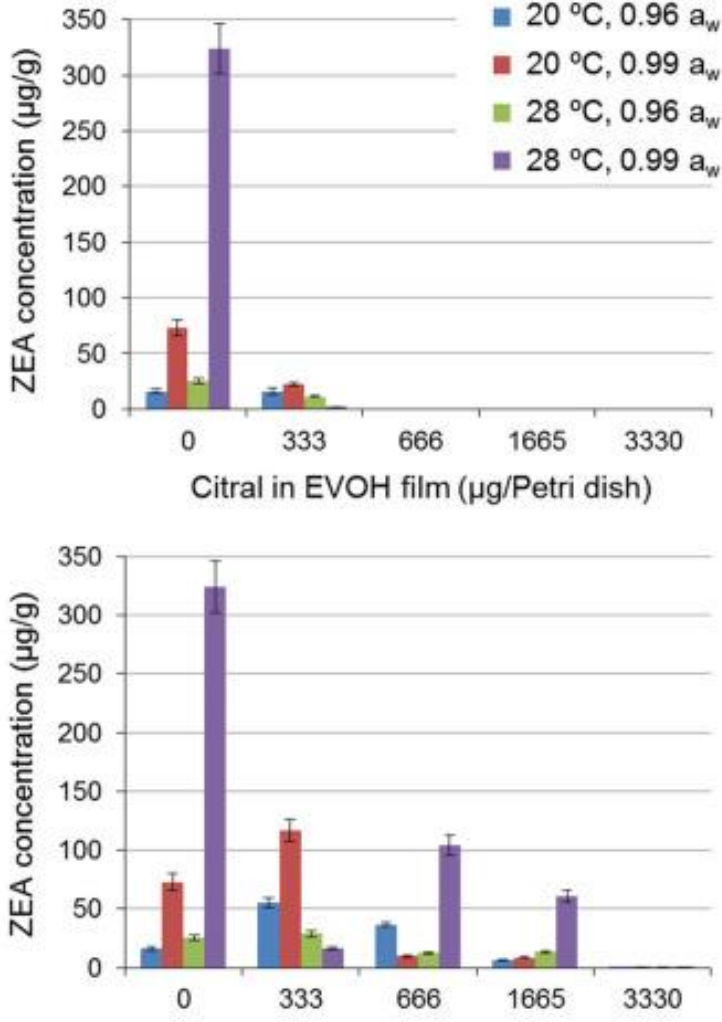

Linalool in EVOH film ( $\mu \mathrm{g} /$ Petri dish)

Fig. 3. Zearalenone production by F. culmorum in cultures on partially milled maize grain incubated with $\mathrm{EVOH}$ films containing cinnamaldehyde, citral, isoeugenol or linalool at different doses in vapor phase under different $a_{w}$ and temperature regimes. Incubation period: 21 days. Error bars represent standard deviations. 

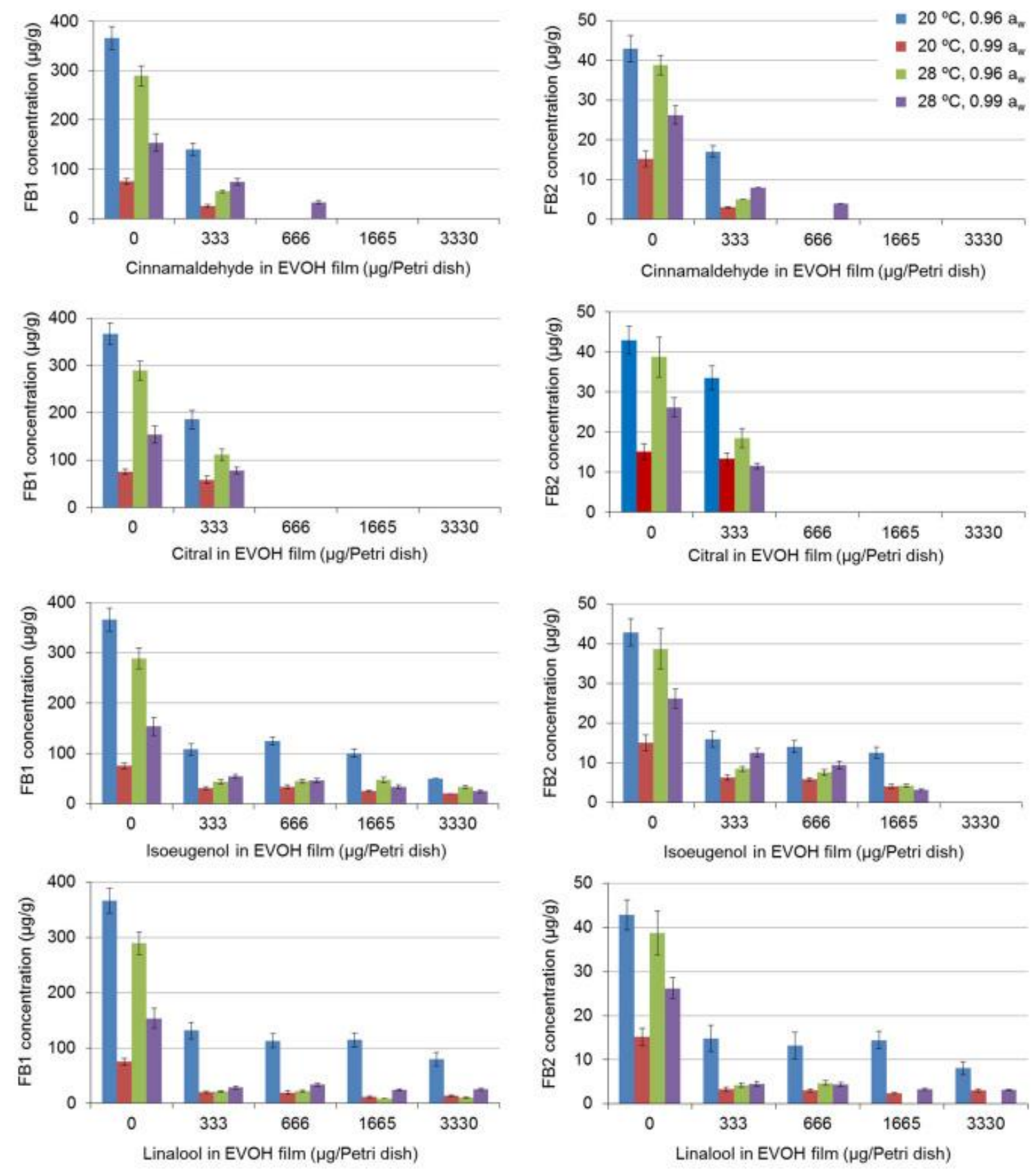

Fig. 4. Fumonisin $B_{1}$ and $B_{2}$ production by $F$. proliferatum in cultures on partially milled maize grain incubated with $\mathrm{EVOH}$ films containing cinnamaldehyde, citral, isoeugenol or linalool at different doses in vapor phase under different $a_{w}$ and temperature regimes. Incubation period: 21 days. Error bars represent standard deviations. 
a) Fusarium culmorum
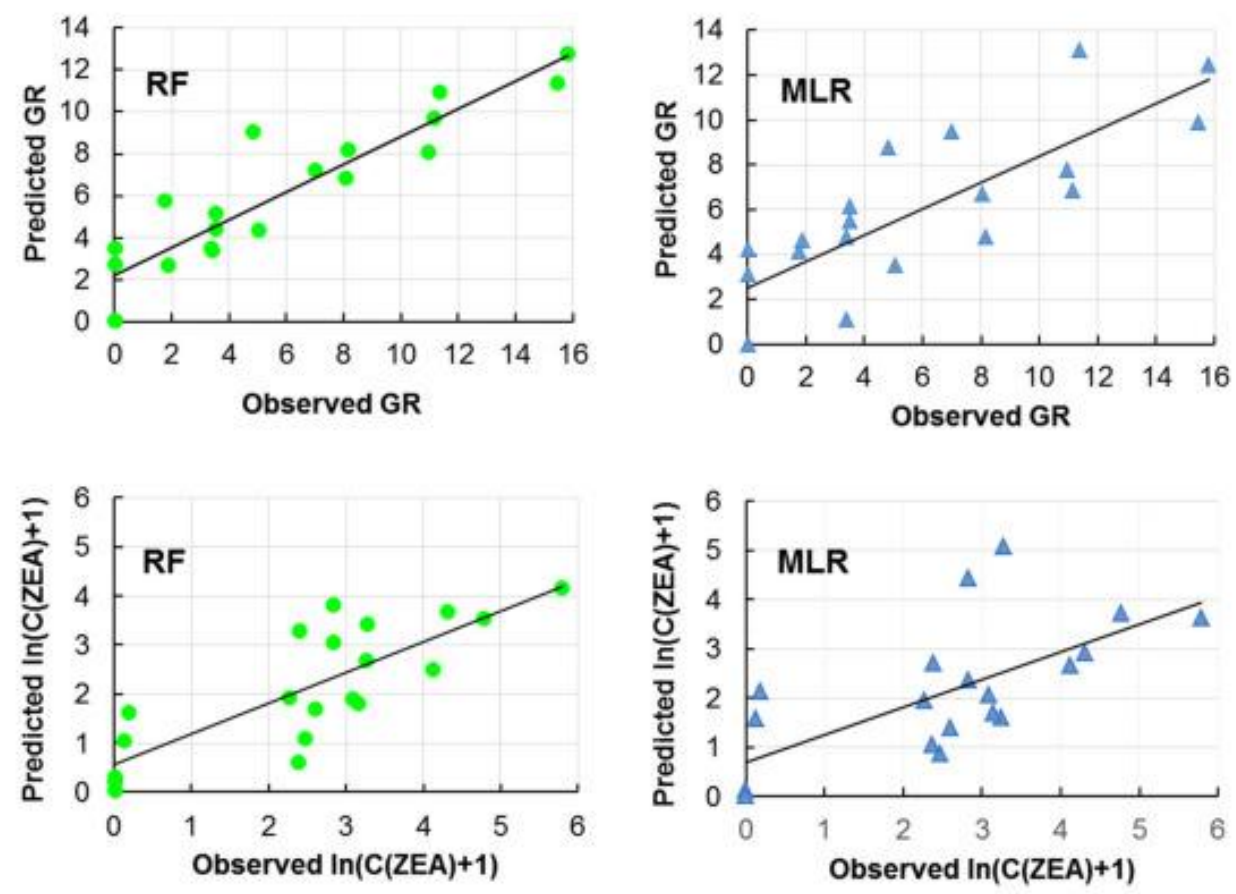

\section{b) Fusarium proliferatum}
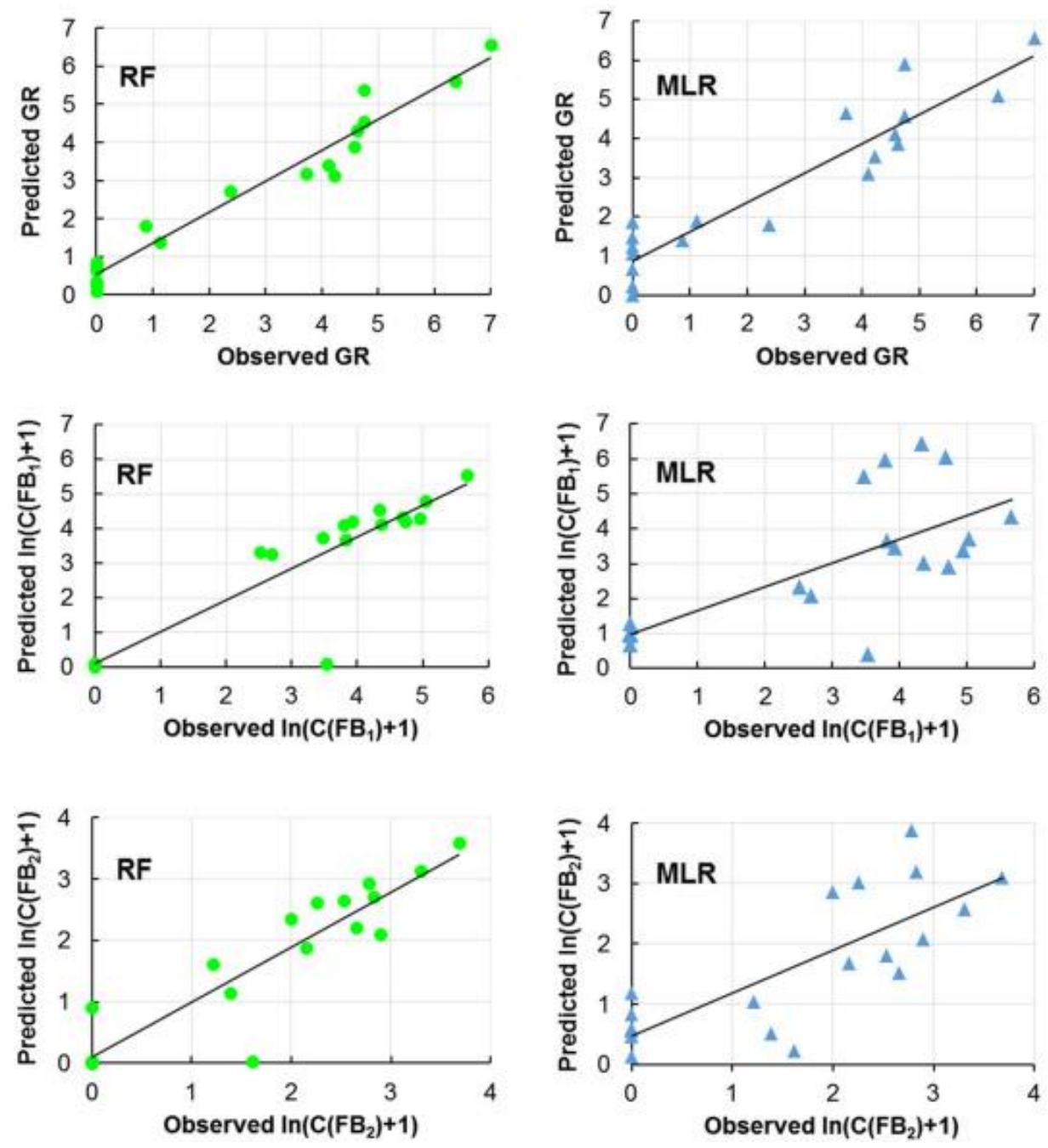
Fig. 5. Scatter plots and lines of best fit of predicted output values provided by the predictive models versus observed (experimental) output values for test datasets. The outcomes were GR and ZEA production for $F$. culmorum and $\mathrm{GR}$ and $\mathrm{FB}_{1} / \mathrm{FB}_{2}$ production for $F$. proliferatum. RF: random forest model; MLR: multiple linear regression model. For mycotoxins, the natural logarithms of concentration plus 1 were computed as output values. 